\title{
Optical phonons for Peierls chains with long-range Coulomb interactions
}

\author{
Máté Timár ${ }^{1,2}$, Gergely Barcza ${ }^{1}$, Florian Gebhard ${ }^{3}$ * and Örs Legeza团 \\ ${ }^{1}$ Strongly Correlated Systems Lendület Research Group, Institute for Solid State Physics and Optics, \\ MTA Wigner Research Centre for Physics, P.O. Box 49, H-1525 Budapest, Hungary \\ ${ }^{2}$ Department of Physics of Complex Systems, Eötvös University, H-1117 Budapest, Hungary and \\ ${ }^{3}$ Fachbereich Physik, Philipps-Universität Marburg, D-35032 Marburg, Germany
}

(Dated: Submitted version of November 14, 2016)

\begin{abstract}
We consider a chain of atoms that are bound together by a harmonic force. Spin- $1 / 2$ electrons that move between neighboring chain sites (Hückel model) induce a lattice dimerization at half band filling (Peierls effect). We supplement the Hückel model with a local Hubbard interaction and a long-range Ohno potential, and calculate the average bond-length, dimerization, and optical phonon frequencies for finite straight and zig-zag chains using the density-matrix renormalization group (DMRG) method. We check our numerical approach against analytic results for the Hückel model. The Hubbard interaction mildly affects the average bond length but substantially enhances the dimerization and increases the optical phonon frequencies whereas, for moderate Coulomb parameters, the long-range Ohno interaction plays no role.
\end{abstract}

\section{INTRODUCTION}

The calculation of lattice vibrations in ordinary metals and band insulators is one of the basic tasks in theoretical solid-state physics. ${ }^{1-5}$ Phonon dispersions can be measured with inelastic neutron scattering, and infrared spectroscopy permit the detection of vibrations with finite energy and vanishingly small momenta ('optical phonons') in crystals and in molecules. ${ }^{9}-11$

For correlated electron systems, however, the calculation of phonon frequencies is still at its beginning. For one-dimensional systems for example, theoretical investigations focus on the ground-state phase diagram of the Holstein-Hubbard model where the competition between the electron-phonon coupling and the electronelectron interaction leads to a rich ground-state phase diagram. $12-16$

In the adiabatic limit where the phonons can be treated classically, one-dimensional electronic systems at half band-filling dimerize (Peierls effect), 17 as is observed in $\pi$-conjugated polymers such as trans-polyacetylene. 18,19 The phonon spectrum of such a Peierls insulator cannot be described by short-range forces ('harmonic springs') acting between atoms at short distances because the optical phonon branch shows a non-trivial momentum dependence with a strong reduction at small momenta (Kohn anomaly) $\stackrel{17}{\prime}$ a Peierls chain of non-interacting electrons provides a well-known example for the Kohn anomaly 20 However, it is not well understood how the electronelectron interaction influences the (optical) phonon frequencies.

In this work, we study electrons that move between neighboring sites on a half-filled chain (Hückel model)21 so that the system describes a Peierls insulator. We add a local Hubbard interaction and a long-range Ohno potential that approximates the electrons' Coulomb interaction (Hückel-Hubbard and Hückel-Hubbard-Ohno models). Reliable numerical investigations of groundstate and excited-state properties for long chains have become possible only recently using the Density-Matrix
Renormalization Group (DMRG) method;22,23 for developments of the method in the last decade, see Refs. [2426] and references therein. We find that the Coulomb interaction suppresses the Kohn anomaly and increases the frequency of the optical phonons.

Our work is structured as follows. In Sect. [II, we set up our Hückel-Hubbard-Ohno Hamiltonian for the itinerant electrons that move over a straight or zig-zag backbone of harmonically bound atoms. Moreover, we define the backbone distortions that correspond to optical phonons.

In Sect. III we analyze the Hückel model for noninteracting electrons analytically for periodic boundary conditions and numerically for open boundary conditions using the DMRG method. For Peierls insulators with a sizable gap, the average bond length, the dimerization, the single-particle gap, and the optical phonon frequencies for systems with up to $L_{C}=110$ sites can safely be extrapolated to the thermodynamic limit.

In Sect. IV, we show that the Hubbard interaction is primarily responsible for the increase of the dimerization and of the phonon frequencies in comparison with the results for the bare Hückel model. A moderately large Ohno interaction leads to very small corrections only. Since the Hubbard interaction substantially increases the single-particle gap, finite-size effects are well under control. For our parameter set, the calculated optical phonon frequencies on a zig-zag chain are in the range of measured values in trans-polyacetylene. ${ }^{27-30}$

Summary and conclusions, Sect. V] close our presentation. Technical details are deferred to two appendices and the supplemental material.

\section{MODEL}

Our model study mimics the properties of transpolyacetylene. We focus on the carbon-carbon stretch and bend modes so that we can work with a small set of parameters. The calculation of optical phonon frequencies in trans-polyacetylene requires a more sophisticated 
description of the structure, namely, the motion of the hydrogen atoms ( $\mathrm{C}-\mathrm{H}$ bond stretching and bending) must be included.

\section{A. Structure}

The carbon atoms in trans-polyacetylene are arranged in a zig-zag chain. For a perfect $\mathrm{sp}^{2}$ hybridization of the carbon $2 s-2 p$ orbitals, the atoms arrange in a zig-zag chain as ground-state conformation with $\vartheta_{l}^{(0)}=\chi_{l}^{(0)}=$ $\varphi_{l}^{(0)}=\Theta_{0}=2 \pi / 3=120^{\circ}$, see Fig. 1. For illustrative purposes and for comparison with earlier work, we shall also address a straight chain with $\vartheta_{l}^{(0)}=\pi=180^{\circ}, \chi_{l}^{(0)}=$ $\varphi_{l}^{(0)}=\pi / 2=90^{\circ}$.

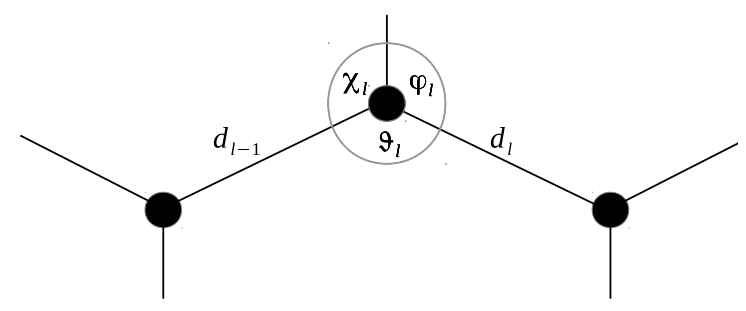

FIG. 1. Coordinates and angles in a planar and unflexed dimerized zig-zag chain.

For our analytic calculations we consider a chain with $L_{C}=2 L$ atoms that is supposed to be planar and unflexed. The atoms occupy the positions $(l=1,2, \ldots 2 L)$

$$
\vec{r}_{l}=\left(\begin{array}{l}
x_{l} \\
y_{l}
\end{array}\right) \text {. }
$$

We denote the ground-state coordinates by capital letters, $\vec{r}_{l}^{(0)} \equiv\left(X_{l}, Y_{l}\right)^{\mathrm{T}}$. We orient the chain to the right of the origin, $X_{1}=Y_{1}=0$ and $X_{3}>0, Y_{3}=0$ in the ground state. For our numerical investigations, we add two atoms, one at the beginning and one at the end of the chain, whose positions are kept fixed during the geometry optimization. In the following we formulate our equations for $L_{C}=2 L$.

\section{B. Contributions to the ground-state energy}

In the ground state, the bond lengths are alternating between long 'single' bonds and short 'double' bonds. The Peierls distortion is due to the itinerant electrons that interact via the Hubbard-Ohno interaction. For the zig-zag chain, a clock spring models the repulsive interaction of the $\sigma$-bonds.

\section{Electronic Hamiltonian}

The system is half filled, i.e., the number of electrons equals the number of sites, $N_{\uparrow}+N_{\downarrow}=L_{C}=2 L$; it is paramagnetic, $N_{\uparrow}=N_{\downarrow}=L_{C} / 2=L$. The electrons move between neighboring sites (Hückel model)21

$$
\hat{T}=-\sum_{\sigma} \sum_{l=1}^{2 L-1} t_{l}\left(\hat{c}_{l+1, \sigma}^{\dagger} \hat{c}_{l, \sigma}+\hat{c}_{l, \sigma}^{\dagger} \hat{c}_{l+1, \sigma}\right),
$$

where $\hat{c}_{l, \sigma}^{\dagger}\left(\hat{c}_{l, \sigma}\right)$ creates (annihilates) an electron with spin $\sigma=\{\uparrow, \downarrow\}$ on carbon atom $l$. The parameters for the electron transfer between nearest neighbors are given by the Peierls expression $(l=1,2, \ldots, 2 L-1)$

$$
t_{l}=t\left(d_{l}\right)=t_{0} \exp \left(-\left(d_{l}-r_{0}\right) \alpha / t_{0}\right)
$$

where $t_{0}=2.5 \mathrm{eV}$ is the electron transfer parameter at distance $r_{0}=1.4 \AA$, and $\alpha=4.0 \mathrm{eV} / \AA$ parameterizes the Peierls coupling. 31 Moreover, the nearest-neighbor distances $d_{l}$ as a function of the coordinates $\left\{x_{l}\right\},\left\{y_{l}\right\}$ are given by

$$
d_{l}=\left|\vec{d}_{l}\right|=\sqrt{\left(x_{l+1}-x_{l}\right)^{2}+\left(y_{l+1}-y_{l}\right)^{2}} .
$$

More generally, we denote the distance between the atoms $i$ and $j$ by

$$
d_{i j}=\left|\vec{r}_{i}-\vec{r}_{j}\right|=\sqrt{\left(x_{i}-x_{j}\right)^{2}+\left(y_{i}-y_{j}\right)^{2}} .
$$

The Coulomb interaction between the electrons is given by the Hubbard-Ohno interaction,

$$
\begin{aligned}
\hat{H}_{\mathrm{int}}= & U \sum_{l=1}^{2 L}\left(\hat{n}_{l, \uparrow}-1 / 2\right)\left(\hat{n}_{l, \downarrow}-1 / 2\right) \\
& +\frac{1}{2 \epsilon_{d}} \sum_{l \neq m=1}^{2 L} V\left(d_{l m}\right)\left(\hat{n}_{l}-1\right)\left(\hat{n}_{m}-1\right),
\end{aligned}
$$

where $\hat{n}_{l, \sigma}=\hat{c}_{l, \sigma}^{\dagger} \hat{c}_{l, \sigma}$ counts the number of $\sigma$-electrons on carbon atom $l$, and $\hat{n}_{l}=\hat{n}_{l, \uparrow}+\hat{n}_{l, \downarrow}$. We parameterize the distance-dependence of the density-density interaction by the Ohno expression 18,19

$$
V(x)=\frac{V}{\sqrt{1+\beta(x / \AA)^{2}}}, \beta=\left(\frac{V}{14.397 \mathrm{eV}}\right)^{2} .
$$

The Ohno form guarantees that, at large distances, the electrons interact via their unscreened Coulomb interaction, $e^{2}=14.397 \mathrm{eV} \AA$. In this study we use the Coulomb and screening parameters $U=6 \mathrm{eV}, V=3 \mathrm{eV}, \epsilon_{d}=2.3$, as in our investigation of polydiacetylene. 32

The Hückel-Hubbard-Ohno model reads

$$
\hat{H}_{\mathrm{el}}=\hat{T}+\hat{H}_{\mathrm{int}}
$$

We must determine the electronic ground-state energy

$$
E_{\mathrm{el}}\left(\left\{x_{l}\right\},\left\{y_{l}\right\}\right)=\left\langle\Psi_{0}\left|\hat{H}_{\mathrm{el}}\right| \Psi_{0}\right\rangle
$$

$E_{\text {el }}$ parametrically depends on the positions of the atoms. For our analytical calculations for the Hückel model we 
use periodic boundary conditions for a ring with $L_{C}=2 L$ atoms, see appendix A.

For our numerical investigations for the Hückel-Hubbard(-Ohno) model, we employ open boundary conditions for chains with $L_{C}=2 L+2$ atoms. The DMRG provides highly accurate results for large system sizes with up to $L_{C}=110$ sites, see appendix B.

\section{Bond compression/stretching energy}

In the adiabatic limit, the energy for bond compression or stretching parametrically depends on the distances between neighboring atoms,

$$
E_{\mathrm{CC}}\left(\left\{x_{l}\right\},\left\{y_{l}\right\}\right)=\sum_{l=1}^{2 L-1} V_{\sigma}\left(d_{l}\right)
$$

We use a linear force function

$$
V_{\sigma}(r)=K_{\sigma, 0}\left(r-r_{0}\right)+\frac{K_{\sigma, 1}}{2}\left(r-r_{0}\right)^{2},
$$

where $r_{0}=1.4 \AA$ is the average carbon atom distance in trans-polyacetylene. For most of our study we use $K_{\sigma, 0}=-4.8 \mathrm{eV} / \AA$ and $K_{\sigma, 1}=42 \mathrm{eV} / \AA^{2}$, as motivated in Ref. 31.

\section{Bond bending energy}

The electronic Hamiltonian and the $\sigma$-bond distortion term do not lead to a zig-zag geometry in the ground state. To stabilize the structure shown in Fig. 1 we include the repulsion of the $\sigma$-bonds via a clock spring,

$$
E_{\mathrm{CC} \mathrm{b}}\left(\left\{x_{l}\right\},\left\{y_{l}\right\}\right)=\frac{C_{\mathrm{b}}}{2} \sum_{l=1}^{2 L-1}\left(\cos \left(\vartheta_{l}\right)-\cos \left(\Theta_{0}\right)\right)^{2}
$$

with

$$
\begin{aligned}
\cos \left(\pi-\vartheta_{l}\right)= & \frac{\left(x_{l+1}-x_{l}\right)\left(x_{l}-x_{l-1}\right)}{d_{l} d_{l-1}} \\
& +\frac{\left(y_{l+1}-y_{l}\right)\left(y_{l}-y_{l-1}\right)}{d_{l} d_{l-1}}
\end{aligned}
$$

For the straight chain, we set $C_{\mathrm{b}}=0$ and arrange all atoms on a line, i.e., we set $y_{l}=0$ from the outset.

To second order in $\left(\vartheta_{l}-\Theta_{0}\right)$, we may equally write

$$
E_{\mathrm{CCb}}\left(\left\{x_{l}\right\},\left\{y_{l}\right\}\right)=\frac{\widetilde{C}_{\mathrm{b}}}{2} \sum_{l=1}^{2 L-1}\left(\vartheta_{l}-\Theta_{0}\right)^{2}
$$

with $\widetilde{C}_{\mathrm{b}}=C_{\mathrm{b}} \sin ^{2}\left(\Theta_{0}\right)$. The clock-spring constants differ by a factor $\sin ^{2}\left(\Theta_{0}\right)=3 / 4$ when we work with angles, as in eq. (14), instead of their cosines, as in eq. (12). For the zig-zag chains, we set $\tilde{C}_{\mathrm{b}}=3.5 \mathrm{eV} / \mathrm{rad}^{2}$, i.e., $C_{\mathrm{b}}=4.667 \mathrm{eV}$, which is a reasonable value for polymers.

\section{Total energy}

The total energy of the structure is the sum of all three contributions. We abbreviate the coordinates of the atoms in the $l$ th unit cell $(l=1, \ldots, L)$ by

$$
\vec{p}_{l}=\left(\begin{array}{c}
x_{2 l} \\
y_{2 l} \\
x_{2 l-1} \\
y_{2 l-1}
\end{array}\right) .
$$

Then, the total energy of the structure reads

$$
\begin{aligned}
E_{\text {struc }}\left(\left\{\vec{p}_{l}\right\}\right)= & E_{\mathrm{el}}\left(\left\{x_{n}\right\},\left\{y_{n}\right\}\right)+E_{\mathrm{CC}}\left(\left\{x_{n}\right\},\left\{y_{n}\right\}\right) \\
& +E_{\mathrm{CC} \mathrm{b}}\left(\left\{x_{l}\right\},\left\{y_{l}\right\}\right) .
\end{aligned}
$$

It must be minimized with respect to the positions of the carbon atoms $\left(\left\{x_{n}\right\},\left\{y_{n}\right\}\right)$,

$$
\begin{aligned}
E_{0} & =E_{\text {struc }}\left(\left\{\vec{R}_{l}\right\}\right), \\
\left.\frac{\partial E_{\text {struc }}\left(\left\{\vec{p}_{l}\right\}\right)}{\partial p_{l, j}}\right|_{\vec{p}_{l}=\vec{R}_{l}} & =0 \quad \text { for } j=1, \ldots, 4 .
\end{aligned}
$$

By construction, we find the minimum $E_{0}$ of the energy functional $E_{\text {struc }}$ at the optimal atomic positions $\vec{R}_{l}=$ $\left(X_{2 l}, Y_{2 l}, X_{2 l-1}, Y_{2 l-1}\right)^{\mathrm{T}}$ for $l=1, \ldots, L$.

\section{Optical phonons}

The second derivatives of the ground-state energy with respect to the atomic positions define the dynamical matrix from which the phonon spectrum can be calculated. $\underline{5}$ In the following we shall focus on distortions that are identical in each unit cell ('optical phonons').

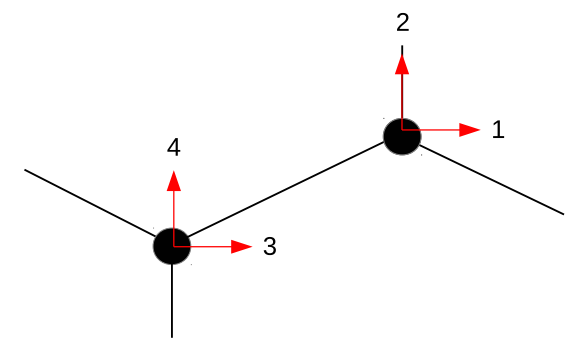

FIG. 2. Four distortions in the unit cell for optical phonons in a planar and unflexed zig-zag chain.

\section{Distortions and dynamical matrix}

By definition, optical phonons are characterized by the fact that the motion of all atoms is the same when going from one unit cell to the next. This results from the fact that the light field adds vanishingly small momentum to the system $\frac{\sqrt{x}}{}$ Thus, we may set

$$
\overrightarrow{p_{l}}-\vec{R}_{l}=\vec{\delta}=\left(\delta_{1}, \delta_{2}, \delta_{3}, \delta_{4}\right)^{\mathrm{T}}
$$


The energy in presence of the distortion becomes a function of the four parameters $\vec{\delta}$,

$$
E(\vec{\delta})=E_{\text {struc }}\left(\left\{\vec{R}_{l}+\vec{\delta}\right\}\right) .
$$

The distortions are shown schematically in Fig. 2.

The chain is symmetric under a rotation by $180^{\circ}$ around its midpoint. It is useful to work with lattice distortions that respect this $C_{2}$-symmetry. Therefore, for our calculations we henceforth use

$$
\begin{aligned}
\vec{p}_{l}-\vec{R}_{l}=\overrightarrow{\tilde{\delta}} & =\left(\tilde{\delta}_{1}, \tilde{\delta}_{2}, \tilde{\delta}_{3}, \tilde{\delta}_{4}\right)^{\mathrm{T}}=(\underline{\underline{O}} \cdot \vec{\delta})^{\mathrm{T}} \\
& =\left(\frac{\delta_{1}+\delta_{3}}{\sqrt{2}}, \frac{\delta_{2}+\delta_{4}}{\sqrt{2}}, \frac{\delta_{1}-\delta_{3}}{\sqrt{2}}, \frac{\delta_{2}-\delta_{4}}{\sqrt{2}}\right)^{\mathrm{T}}
\end{aligned}
$$

with

$$
\underline{\underline{O}}^{+}=\underline{\underline{O}}^{-1}=\underline{\underline{O}}=\sqrt{\frac{1}{2}}\left(\begin{array}{rrrr}
1 & 0 & 1 & 0 \\
0 & 1 & 0 & 1 \\
1 & 0 & -1 & 0 \\
0 & 1 & 0 & -1
\end{array}\right)
$$

to separate the symmetry sectors $A_{g}$ ('gerade') and $B_{u}$ ('ungerade').

For the calculation of the dynamical matrix, we need to Taylor expand the energy to second order,

$$
E(\vec{\delta}) \approx E_{0}+\frac{L}{2} \sum_{i, j=1}^{4} K_{i, j} \delta_{i} \delta_{j}
$$

where we used eqs. (17) and (18) and defined the elements of the real, symmetric dynamical matrix

$$
K_{i, j}=\left.\frac{1}{L} \sum_{n, m=1}^{L} \frac{\partial^{2} E_{\text {struc }}\left(\left\{\vec{p}_{l}\right\}\right)}{\partial p_{n ; i} \partial p_{m ; j}}\right|_{\vec{p}_{l}=\vec{R}_{l}}
$$

for optical phonons. Correspondingly, we have

$$
E(\vec{\delta}) \approx E_{0}+\frac{L}{2} \sum_{i, j=1}^{4} \tilde{K}_{i, j} \tilde{\delta}_{i} \tilde{\delta}_{j}
$$

with $\underline{\underline{\tilde{K}}}=\underline{\underline{O}}^{+} \cdot \underline{\underline{K}} \cdot \underline{\underline{O}}$. Note that $\underline{\underline{\underline{K}}}$ is block diagonal,

$$
\underline{\underline{\tilde{K}}}=\left(\begin{array}{cc}
\underline{\underline{B_{u}}} & \underline{\underline{\underline{\underline{A}}}} \\
\underline{\underline{\underline{A_{g}}}} & \underline{\underline{\underline{A_{g}}}}
\end{array}\right)
$$

where $\underline{\underline{A_{g}}}$ and $\underline{\underline{B_{u}}}$, are $2 \times 2$ matrices and $\underline{\underline{0}}$ is the $2 \times 2$ zero matrix.

\section{Classical Hamilton function and phonon frequencies}

The corresponding classical Hamilton function for the displacement in one unit cell is given by

$$
H_{\mathrm{ph}}\left(\left\{\dot{\delta}_{l}\right\},\left\{\delta_{l}\right\}\right)=T\left(\left\{\dot{\delta}_{l}\right\}\right)+V\left(\left\{\delta_{l}\right\}\right)
$$

with

$$
\begin{aligned}
& T\left(\left\{\dot{\delta}_{l}\right\}\right)=\frac{M}{2} \sum_{i=1}^{4}\left(\dot{\delta}_{i}\right)^{2}=\frac{M}{2} \sum_{i=1}^{4}\left(\dot{\tilde{\delta}}_{i}\right)^{2}, \\
& V\left(\left\{\delta_{l}\right\}\right)=\frac{1}{2} \sum_{i, j=1}^{4} K_{i, j} \delta_{i} \delta_{j}=\frac{1}{2} \sum_{i, j=1}^{4} \tilde{K}_{i, j} \tilde{\delta}_{i} \tilde{\delta}_{j},
\end{aligned}
$$

where $M$ is the mass of the atoms.

The optical phonon frequencies can be derived from

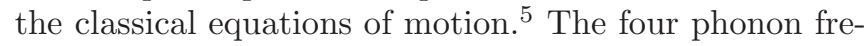
quencies result from the zeros of the characteristic polynomials $P_{A, B}\left(\omega^{2}\right)$ with

$$
\begin{aligned}
& P_{A}\left(\omega^{2}\right) \equiv \operatorname{Det}\left(-\omega^{2} M \underline{\underline{1}}+\underline{\underline{A_{g}}}\right)=0, \\
& P_{B}\left(\omega^{2}\right) \equiv \operatorname{Det}\left(-\omega^{2} M \underline{\underline{1}}+\underline{\underline{B_{u}}}\right)=0,
\end{aligned}
$$

where $M=12 u\left(1 u=1.66054 \cdot 10^{-27} \mathrm{~kg}\right)$ is the mass of an atom and $\underline{\underline{1}}$ is the $2 \times 2$ unit matrix. Since all atoms have equal mass, we immediately find $(l=1,2)$

$$
\omega_{l}^{\mathrm{acc}}=\sqrt{\frac{\tilde{K}_{B, l}}{M}} \quad, \quad \omega_{l}^{\mathrm{opt}}=\sqrt{\frac{\tilde{K}_{A, l}}{M}},
$$

where $\tilde{K}_{A / B, l}$ are the two eigenvalues of the dynamical matrices $\underline{\underline{A_{g}}}$ and $\underline{\underline{B_{u}}}$, respectively.

For periodic boundary conditions, the two eigenvalues in the $B_{u}$ symmetry sector are zero, $\tilde{K}_{B, 1}=\tilde{K}_{B, 2}=0$, because they correspond to a horizontal or vertical motion of the whole chain. For our numerical investigations, we fix the first and last atom so that $\tilde{K}_{B, 1}$ and $\tilde{K}_{B, 2}$ are not exactly zero. This gives rise to two 'acoustic' phonons in our DMRG calculations. Their energies are proportional to the inverse of the total mass of the chain so that the energy of the acoustic modes vanishes in the thermodynamic limit, $\omega_{l}^{\text {acc }} \sim \sqrt{1 / L_{C}}$. Differences between the analytic and numerical results for $\omega_{1}^{\text {opt }}$ and $\omega_{2}^{\mathrm{opt}}$ in the Hückel model can be used to assess the importance of finite-size effects and to test the numerical accuracy of our approach.

When we measure energies in $\mathrm{eV}$ and distances in $\AA$, the entries of the dynamical matrix have the unit $\mathrm{eV} / \AA^{2}$. Thus, the phonon frequencies in eq. (30) are given in units of $\sqrt{\mathrm{eV} / u} / \AA$. To express the phonon frequencies in terms of wavenumbers $\left(\mathrm{cm}^{-1}\right)$, we use the conversion factor

$$
\frac{\lambda^{-1}}{\mathrm{~cm}^{-1}}=\frac{\omega}{\sqrt{\mathrm{eV} / u} / \AA} \sqrt{\frac{1.60219}{1.66054}} \cdot 10^{14} \frac{1}{2 \pi 2.997925 \cdot 10^{10}} .
$$

The conversion factor amounts to 521.473.

\section{HÜCKEL MODEL}

We start the presentation of our results with an analysis of the Hückel model that can be solved analytically for 
periodic boundary conditions. Therefore, we can assess the quality of the numerical DMRG calculations, i.e., we study edge effects and finite-size effects for the average bond length and the dimerization, and the system-size dependence of the optical phonon frequencies. We shall show that DMRG provides a reliable description for finite systems, and we can safely extrapolate the optical phonon data to the thermodynamic limit if the singleparticle gap is converged for the maximal system sizes that we have reached numerically.

\section{A. Average bond length and dimerization}

For non-interacting electrons, the average bond length and dimerization are the same for the straight and zigzag chains because the energy is solely a function of the bond lengths so that bond angles are irrelevant.

\section{Edge effects}

In Fig. 3 we show the bond length $d_{l}$ as a function of the bond coordinate $l$ for the Hückel model on a straight chain with $L=32$ unit cells for periodic boundary conditions (analytic result, pbc) and for open boundary conditions (DMRG, obc). As seen from the figure, the lengths of the single and double bonds obtained from open boundary conditions agree within a small error margin with the analytical result for periodic boundary conditions not only in the center of the chain but for all sites $20<l<46$. Therefore, our calculated average length $\bar{r}$ and dimerization $\Delta$, taken at the middle of the chain, are not influenced by edge effects for moderately large chains, $L \gtrsim 30$.

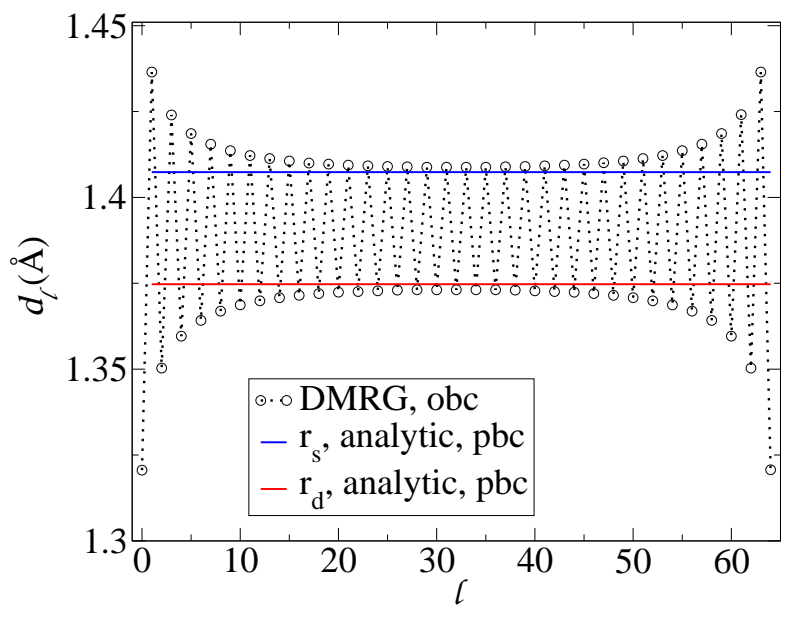

FIG. 3. Bond lengths for the Hückel model on a straight chain with $t_{0}=2.5 \mathrm{eV}, \alpha=4.0 \mathrm{eV} / \AA, K_{\sigma, 0}=-4.8 \mathrm{eV} / \AA$, and $K_{\sigma, 1}=42 \mathrm{eV} / \AA^{2}$ for $L=32$ unit cells for periodic boundary conditions (pbc, analytic calculation, $L_{C}=64$ ) and open boundary conditions (obc, DMRG, $L_{C}=66$ ).

\section{Finite-size effects}

The finite-size extrapolation for the average bond length and the dimerization can nicely be carried out from our DMRG data for up to $L_{C}=110$, see Figs. 4. The average bond length is almost independent of system size and boundary conditions, as seen from the comparison of periodic boundary conditions (analytical result) and open boundary conditions (DMRG).

Finite-size and interaction effects are more pronounced for the dimerization. For the Hückel model, open boundary conditions lead to a larger dimerization than periodic boundary conditions. In both cases, the data for finite system sizes can reliably be extrapolated to the thermodynamic limit even for $K_{\sigma, 1}=42 \mathrm{eV} / \AA^{2}$ that leads to a small dimerization, $\Delta \approx 0.015 \AA$.
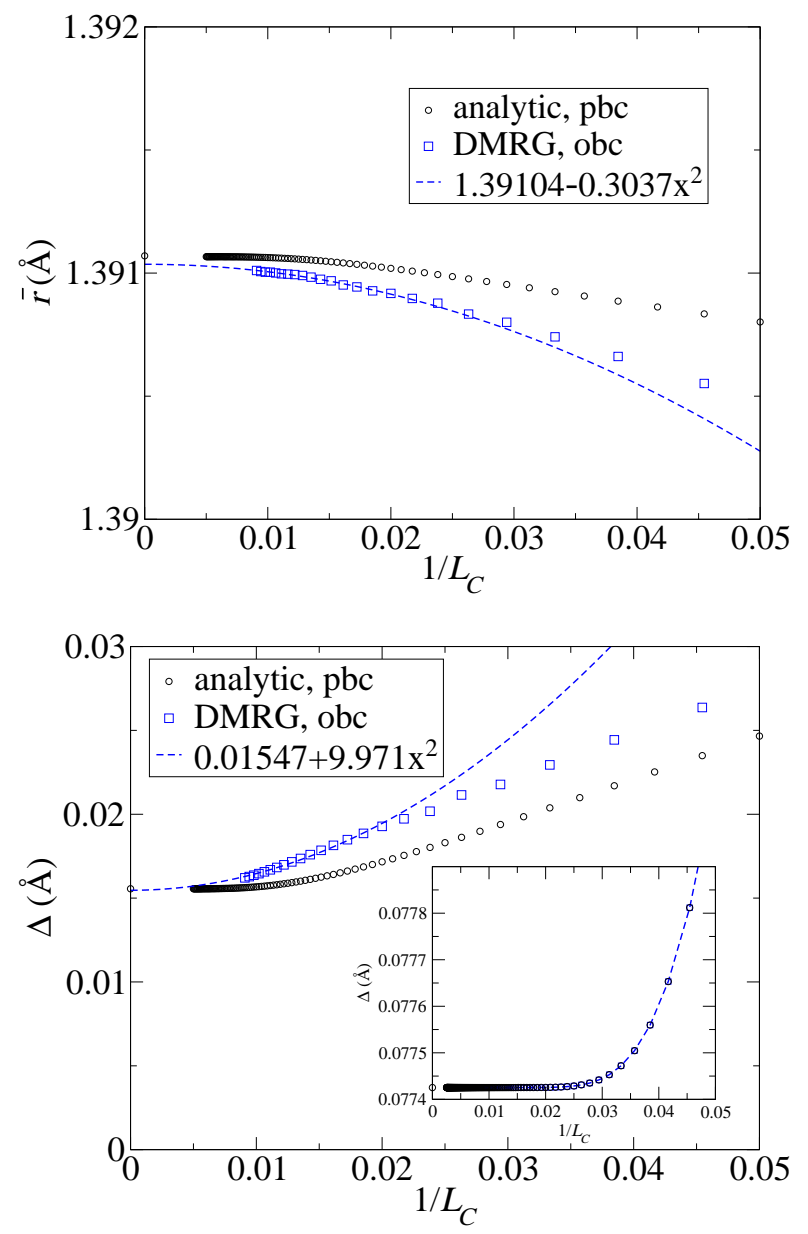

FIG. 4. Average bond length and dimerization for the Hückel model with $t_{0}=2.5 \mathrm{eV}, \alpha=4.0 \mathrm{eV} / \AA, K_{\sigma, 0}=-4.8 \mathrm{eV} / \AA$, and $K_{\sigma, 1}=42 \mathrm{eV} / \AA^{2}$ as a function of the inverse chain length for periodic boundary conditions (pbc, analytic calculation, $L_{C}=2 L$ ) and for open boundary conditions (obc, DMRG, $\left.L_{C}=2 L+2\right)$. The limiting values for the Hückel model are $\bar{r}^{\mathrm{H}}=1.39107 \AA$ and $\Delta^{\mathrm{H}}=0.01555 \AA$. For the quadratic extrapolation of the DMRG data, system sizes $L_{C} \geq 50$ are used. Inset: Dimerization for $K_{\sigma, 1}=29.5 \mathrm{eV} / \AA^{2}$. 
In the inset of Fig. 4 we show the dimerization for $K_{\sigma, 1}=29.5 \mathrm{eV} / \AA^{2}$ that leads to a large dimerization, $\Delta \approx 0.077 \AA$. The convergence is significantly faster, and systems as small as $L_{C}=32$ provide a reliable estimate for the value in the thermodynamic limit.

\section{B. Optical phonons}

The frequency of the optical phonons sensitively depends on the size of the gap. To elucidate this effect, we analyze two different parameter sets.

\section{Large Peierls gap}

We start with a parameter set that leads to a sizable gap and a large dimerization with weak finite-size dependencies. For $K_{\sigma, 1}=29.5 \mathrm{eV} / \AA^{2}$, the average bond length is $\bar{r} \approx 1.38 \AA$, and the dimerization is $\Delta \approx 0.08 \AA$, see the inset of Fig. 4, close to the values used by Su, Schrieffer, and Heeger ${ }^{33}$ by

For the Hückel model the single-particle gap is given

$$
E_{\mathrm{sp}}=2[t(\bar{r}-\Delta)-t(\bar{r}+\Delta)] .
$$

The finite-size dependence of the gap is shown in Fig. 5 .

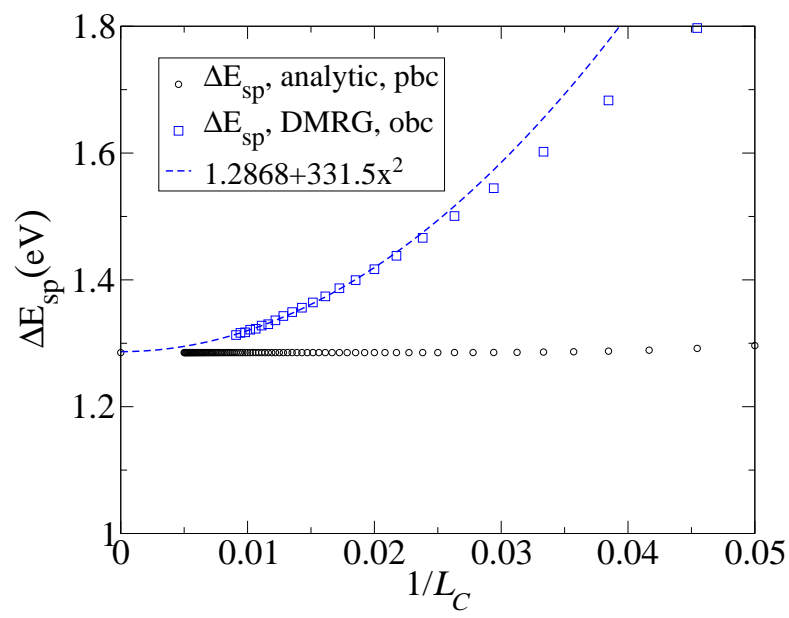

FIG. 5. Single-particle gap for the Hückel model with $t_{0}=2.5 \mathrm{eV}, \alpha=4.0 \mathrm{eV} / \AA, K_{\sigma, 0}=-4.8 \mathrm{eV} / \AA$, and $K_{\sigma, 1}=29.5 \mathrm{eV} / \AA^{2}$ as a function of the inverse chain length for periodic boundary conditions (pbc, analytic calculation, $L_{C}=2 L$ ) and for open boundary conditions (obc, DMRG, $\left.L_{C}=2 L+2\right)$. The limiting value is $\Delta E_{\mathrm{sp}}=1.2853 \mathrm{eV}$. For the quadratic extrapolation of the DMRG data, system sizes $L_{C} \geq 50$ are used.

For a large Peierls gap, the finite-size effects are seen to be small for periodic boundary conditions. The gap value changes by only $1 \%$ from $L_{C}=20$ to the thermodynamic limit. The gap is essentially converged for $L_{C} \gtrsim 50$. For open boundary conditions, however, there are noticeable

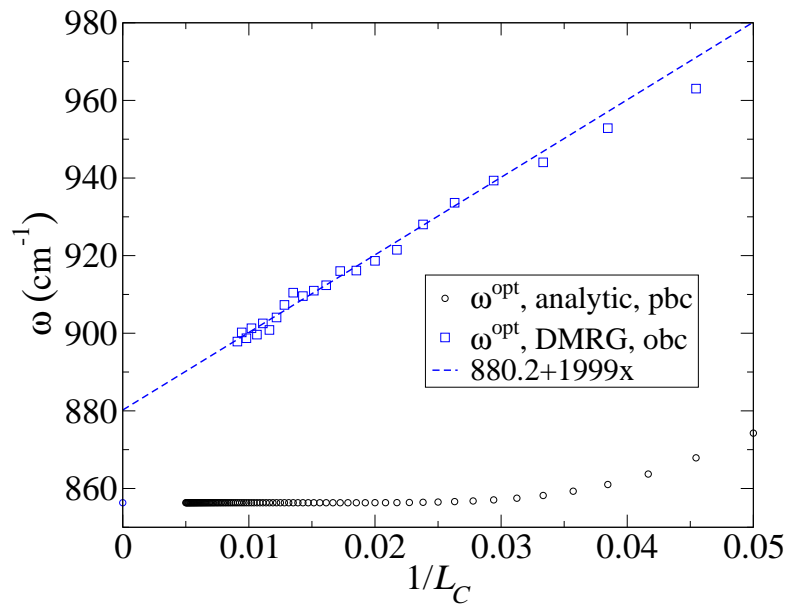

FIG. 6. Optical phonon frequency for the Hückel model on a straight chain as a function of the inverse chain length for the same parameter set as in Fig. 5. The limiting value is $\omega^{\text {opt }}=856.3 \mathrm{~cm}^{-1}$. For the linear extrapolation of the DMRG data, system sizes $L_{C} \geq 50$ are used.

finite-size effects. Systems as large as $L_{C}=100$ are required to detect the quadratic convergence of the gap as a function of $1 / L_{C}$. The extrapolated value agrees with the analytic result with an accuracy of $0.1 \%$.

In Fig. 6] we show the phonon frequency for a straight chain as a function of inverse system size. The finitesize effects are seen to be small for periodic boundary conditions. The phonon frequency changes only by $2 \%$ from $L_{C}=20$ to the thermodynamic limit. For $L_{C} \gtrsim$ 50 , the optical phonon frequency is essentially converged, as also observed for the single-particle gap. For open boundary conditions on the contrary, finite-size effects are substantial. Even for $L_{C}=110$, the optical phonon frequencies show a fairly linear dependence on the inverse system size, and a quadratic behavior on $1 / L_{C}$ is not yet discernible. As a result, a linear extrapolation of the data to the thermodynamic limit gives $\omega_{\infty}^{\text {opt }}=880 \mathrm{~cm}^{-1}$, about $24 \mathrm{~cm}^{-1}$ or $2.8 \%$ larger than the analytic result $\omega^{\text {opt }}=856.3 \mathrm{~cm}^{-1}$ for an infinitely long chain.

We note that the bare optical frequency for a chain without electron-phonon coupling $(\alpha=0)$ is given by $\omega_{0}=\sqrt{4 K_{\sigma, 1} / M}=1635 \mathrm{~cm}^{-1} \underline{20}$ For the Hückel model, we see a strong renormalization of the optical phonon frequency. The renormalization of the phonon frequency is quite large, about a factor of two, $\omega^{\mathrm{opt}} / \omega_{0}=0.524$. This behavior reflects the well-known Kohn anomaly, 18,20,34

In Fig. 7 we show the phonon frequencies for a zigzag chain as a function of inverse system size. The lower (higher) phonon frequency is associated with anti-phase stretching (swinging) of the carbon atoms with respective eigenvectors

$$
\begin{aligned}
& \sqrt{2}\left(\vec{\delta}_{1}^{\text {opt }}\right)^{\mathrm{T}}=(\cos (\gamma), \sin (\gamma),-\cos (\gamma),-\sin (\gamma)) \\
& \sqrt{2}\left(\vec{\delta}_{2}^{\text {opt }}\right)^{\mathrm{T}}=(\sin (\gamma),-\cos (\gamma),-\sin (\gamma), \cos (\gamma)) .
\end{aligned}
$$




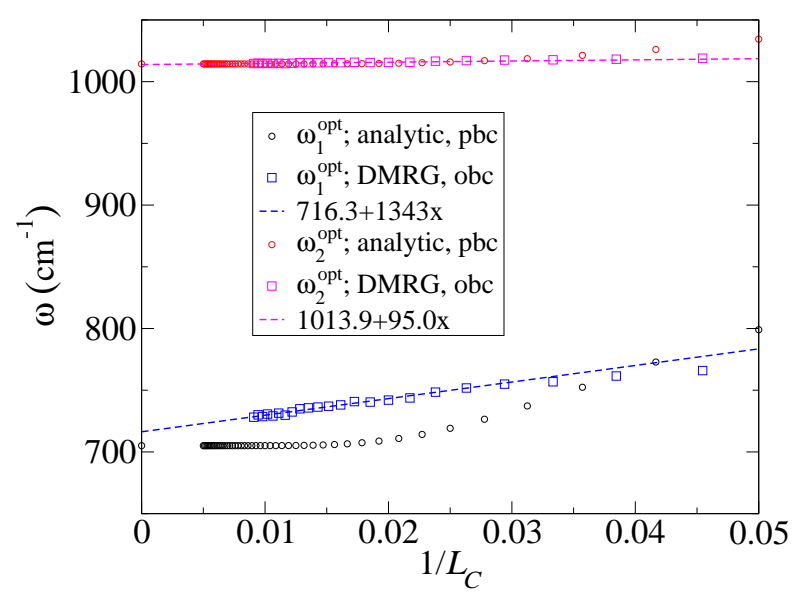

FIG. 7. Optical phonon frequencies for the Hückel model on a zig-zag chain with $\tilde{C}_{b}=3.5 \mathrm{eV} / \mathrm{rad}^{2}$ as a function of the inverse chain length for the same parameter set as in Fig. 5. The limiting values are $\omega_{1}^{\text {opt }}=705.1 \mathrm{~cm}^{-1}$ and $\omega_{2}^{\text {opt }}=1014 \mathrm{~cm}^{-1}$. For the linear extrapolation of the DMRG data, system sizes $L_{C} \geq 50$ are used.

For $L_{C}=80$ sites and periodic boundary conditions we find $\gamma_{\mathrm{H}}=0.355 \mathrm{rad}=20.4^{\circ}$. They result from the diagonalization of the dynamical matrix with the entries $\tilde{K}_{33}^{\mathrm{pbc}}=24.78, \tilde{K}_{34}^{\mathrm{pbc}}=\tilde{K}_{43}^{\mathrm{pbc}}=-7.64$, and $\tilde{K}_{44}^{\mathrm{pbc}}=42.57$ (in units of $\mathrm{eV} / \AA^{2}$ ). The distortions are shown in Fig. 8 The corresponding numbers for open boundary conditions are $\tilde{K}_{33}^{\mathrm{obc}}=27.48, \tilde{K}_{34}^{\mathrm{obc}}=\tilde{K}_{43}^{\mathrm{obc}}=-8.28, \tilde{K}_{44}^{\mathrm{obc}}=$ 41.64 (in units of $\mathrm{eV} / \AA^{2}$ ), and $\gamma_{\mathrm{H}}^{\mathrm{obc}}=0.431 \mathrm{rad}=24.7^{\circ}$.

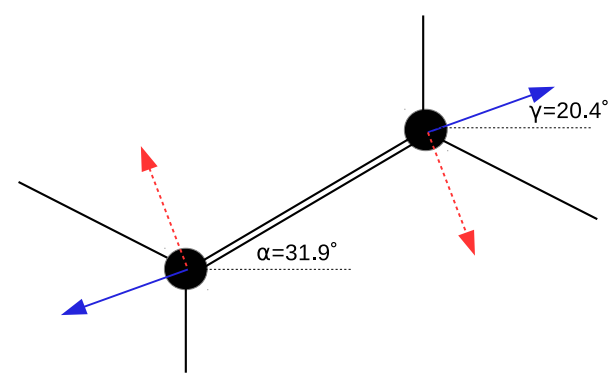

FIG. 8. Optical phonon distortions associated with the antiphase stretching mode at frequency $\omega_{1}^{\text {opt }}$ (blue arrows) and with the anti-phase swinging mode at frequency $\omega_{2}^{\text {opt }}$ (red dotted arrows) for the Hückel model on $L_{C}=80$ sites and periodic boundary conditions. for the same parameter set as in Fig. 5 .

The anti-phase mode at frequency $\omega_{1}^{\mathrm{opt}}$ corresponds to a stretching of the double bond. Therefore, it is strongly linked to the $\pi$-electron system and, correspondingly, it is sensitive to the choice of boundary conditions and to the actual size of the single-particle gap, as already seen for the phonon in the straight chain. Consequently, the linear extrapolation of the DMRG data to the thermodynamic limit in Fig. 7 overestimates the analytic value by about $1.5 \%$.

The anti-phase swinging mode at frequency $\omega_{2}^{\text {opt }}$ in- creases the bond-bending energy but barely changes the kinetic energy of the $\pi$-electrons because the bond lengths remain almost constant. Since $\omega_{2}^{\mathrm{opt}}$ is a very well localized excitation it shows small finite-size effects for both periodic and open boundary conditions. Correspondingly, we recover the analytic value in the thermodynamic limit from a linear extrapolation of the DMRG data with an accuracy of $0.01 \%$, see Fig. 7 .

In sum, for Peierls insulators with a sizable gap DMRG calculations for open boundary conditions can be used to calculate reliably bond lengths, gaps, and optical phonon frequencies in the thermodynamic limit.

\section{Small Peierls gap}

For Peierls insulators with a small single-particle gap, finite-size effects are much larger and it is much more difficult to extract optical phonon frequencies in the thermodynamic limit from finite-size data. To illustrate this feature, we analyze a parameter set that leads to a small dimerization and a small Peierls gap. For $K_{\sigma, 1}=42 \mathrm{eV} / \AA^{2}$, the average bond length is $\bar{r} \approx 1.39 \AA$, and the dimerization is $\Delta \approx 0.016 \AA$, see Fig. 4. The finite-size dependence of the gap is shown in Fig. 9

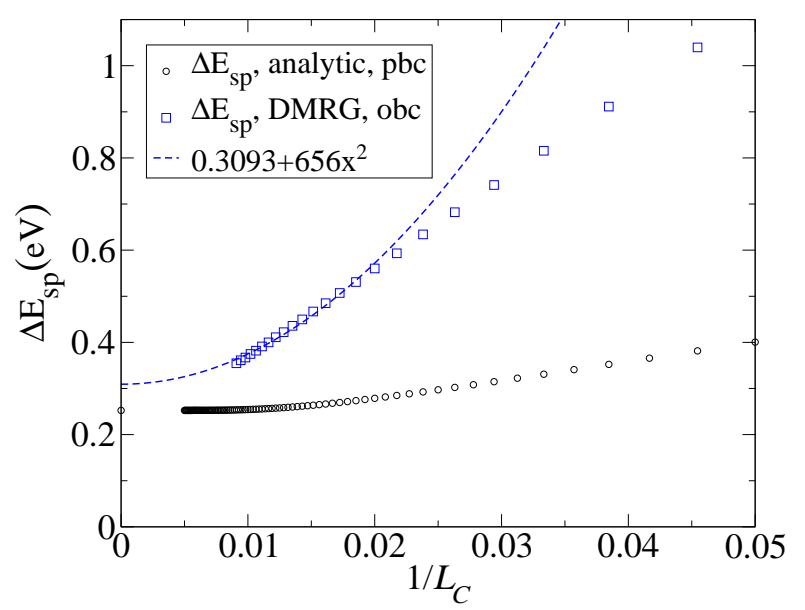

FIG. 9. Single-particle gap for the Hückel model with $t_{0}=$ $2.5 \mathrm{eV}, \alpha=4.0 \mathrm{eV} / \AA, K_{\sigma, 0}=-4.8 \mathrm{eV} / \AA$, and $K_{\sigma, 1}=$ $42 \mathrm{eV} / \AA^{2}$ as a function of the inverse chain length for periodic boundary conditions (pbc, analytic calculation, $L_{C}=2 L$ ) and for open boundary conditions (obc, DMRG, $L_{C}=2 L+2$ ). The limiting value is $\Delta E_{\mathrm{sp}}=0.2525 \mathrm{eV}$. For the quadratic extrapolation of the DMRG data, system sizes $L_{C} \geq 50$ are used.

For a small Peierls gap, the finite-size effects are seen to be relatively large. Even for periodic boundary conditions, the gap value for $L_{C}=20$ is larger than that in the thermodynamic limit by almost a factor two. Concomitantly, the finite-size dependence of the gap is quite large for open boundary conditions. A quadratic dependence of $E_{\mathrm{sp}}\left(L_{C}\right)$ on the inverse system size $1 / L_{C}$ becomes discernible only for $L_{C} \gtrsim 100$. As a consequence, the ex- 


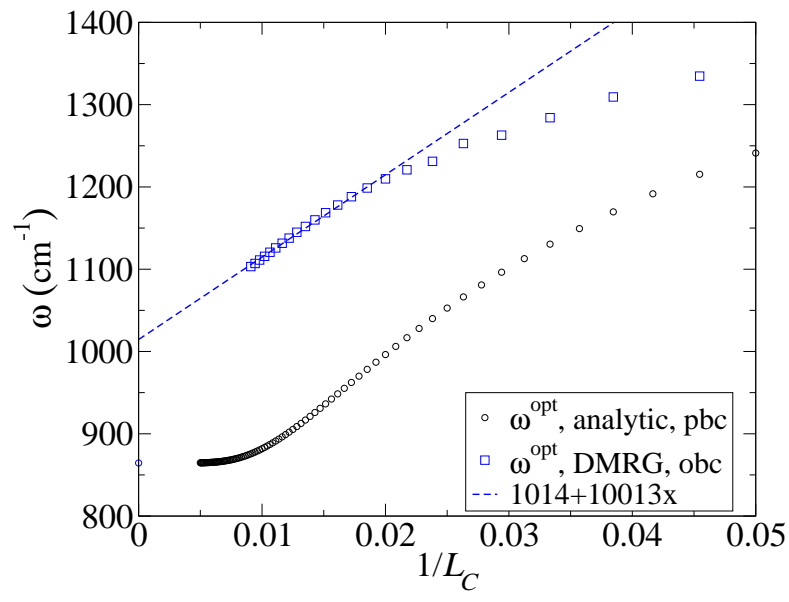

FIG. 10. Phonon frequency for the Hückel model on a straight chain as a function of inverse chain length for the same parameter set as in Fig. 9. The limiting value for the Hückel model is $\omega^{\text {opt }}=864.5 \mathrm{~cm}^{-1}$. For the linear extrapolation of the DMRG data, system sizes $L_{C} \geq 50$ are used.

trapolated gap is $22 \%$ larger than the analytic value in the thermodynamic limit.

In Fig. 10 we show the optical phonon frequency for a straight chain as a function of inverse system size. The finite-size effects are seen to be large even for periodic boundary conditions. The phonon frequency at $L_{C}=20$ is $45 \%$ larger than its value in the thermodynamic limit. A quadratic dependence of $\omega^{\mathrm{opt}}\left(L_{C}\right)$ on the inverse system size $1 / L_{C}$ becomes discernible only for fairly large systems, $L_{C} \gtrsim 100$. Corresponding to the large finite-size effects, the boundary conditions also matter. The analytic results for periodic boundary conditions and the numerical DMRG results for open boundary conditions differ by $250 \mathrm{~cm}^{-1}$ for moderately long chains, $L_{C} \approx 100$. Consequently, the linear extrapolation of the DMRG data to the thermodynamic limit leads to an optical phonon frequency that is $17 \%$ or $150 \mathrm{~cm}^{-1}$ higher than the analytic value.

In Fig. 11 we show the optical phonon frequencies for a zig-zag chain as a function of inverse system size. For short chains, the frequency $\omega_{1}^{\text {opt }}$ of the lower-energy optical phonon is almost independent of $L_{C}$ until its frequency becomes comparable to that of the higher-energy phonon at $\omega_{2}^{\text {opt }}$ which displays a large finite-size renormalization. Then, an avoided crossing occurs around $L_{C} \approx 30$ for our parameter set. The frequency $\omega_{2}^{\mathrm{opt}}$ of the higher-energy phonon levels off and becomes independent of the system size and the choice of boundary conditions. In contrast, the frequency $\omega_{1}^{\mathrm{opt}}$ of the lowerenergy phonon displays large finite-size effects, similarly to the optical phonon of the straight chain. As can be seen from Fig. 11, it drops by almost $350 \mathrm{~cm}^{-1}$ from $L_{C}=20$ to the thermodynamic limit. It requires very large systems to determine $\omega_{1}^{\mathrm{opt}}$ from finite-size extrapolations.

The example shows that the Kohn anomaly is clearly

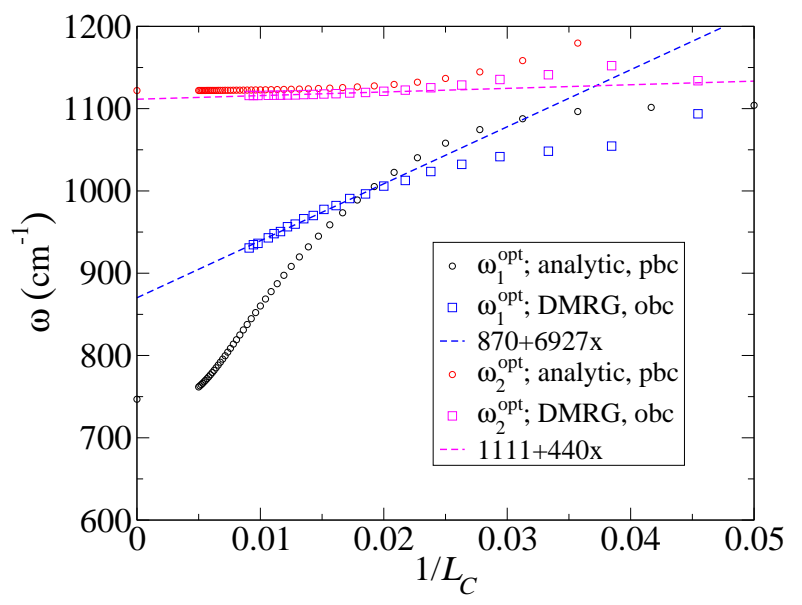

FIG. 11. Optical phonon frequencies for the Hückel model on a zig-zag chain with $\tilde{C}_{b}=3.5 \mathrm{eV} / \mathrm{rad}^{2}$ as a function of the inverse chain length for the same parameter set as in Fig. 9] The limiting values are $\omega_{1}^{\text {opt }}=746.9 \mathrm{~cm}^{-1}$ and $\omega_{2}^{\text {opt }}=1122 \mathrm{~cm}^{-1}$. For the linear extrapolation of the DMRG data, system sizes $L_{C} \geq 50$ are used.

visible in Peierls insulators with a small gap. The dimerization and single-particle gap display large finite-size effects. Correspondingly, the optical phonon frequencies show a substantial finite-size dependence. In the case of the zig-zag chain, an avoided crossing as a function of system size can be seen. However, the Kohn anomaly is suppressed in one-dimensional Mott-Peierls insulators with a sizable single-particle gap, as we shall show in the next section.

\section{HÜCKEL-HUBBARD-OHNO MODEL}

In this section we include the Coulomb interaction. We choose $U=6 \mathrm{eV}$ to obtain the average bond length, dimerization, and single-particle gap as observed for trans-polyacetylene. Moreover, the long-range Ohno interaction with $V=3 \mathrm{eV}$ and static screening parameter $\epsilon_{d}=2.3$ permit us to reproduce the singlet-exciton binding energy 31 The Coulomb interaction increases the frequency of the optical phonons and, due to the large single-particle gap, it eliminates the signatures of the Kohn anomaly as seen for small-gap Peierls insulators in the previous section.

\section{A. Average bond length and dimerization}

For the Hückel-Hubbard(-Ohno) model, finite-size and edge effects are effectively suppressed because the singleparticle gap is large in the presence of the Coulomb interaction. Therefore, the corresponding length scales for the decay of the edge effects are shorter than for the Hückel model, and extrapolations for the average bond length and dimerization are even more robust. 
For non-interacting electrons and for the Hückel-Hubbard model with a purely local interaction, the average bond length and dimerization are the same for the straight and zig-zag chains because the energy is solely a function of the bond lengths so that bond angles are irrelevant. For this reason we do not discriminate the chain geometry for the Hückel-Hubbard model.
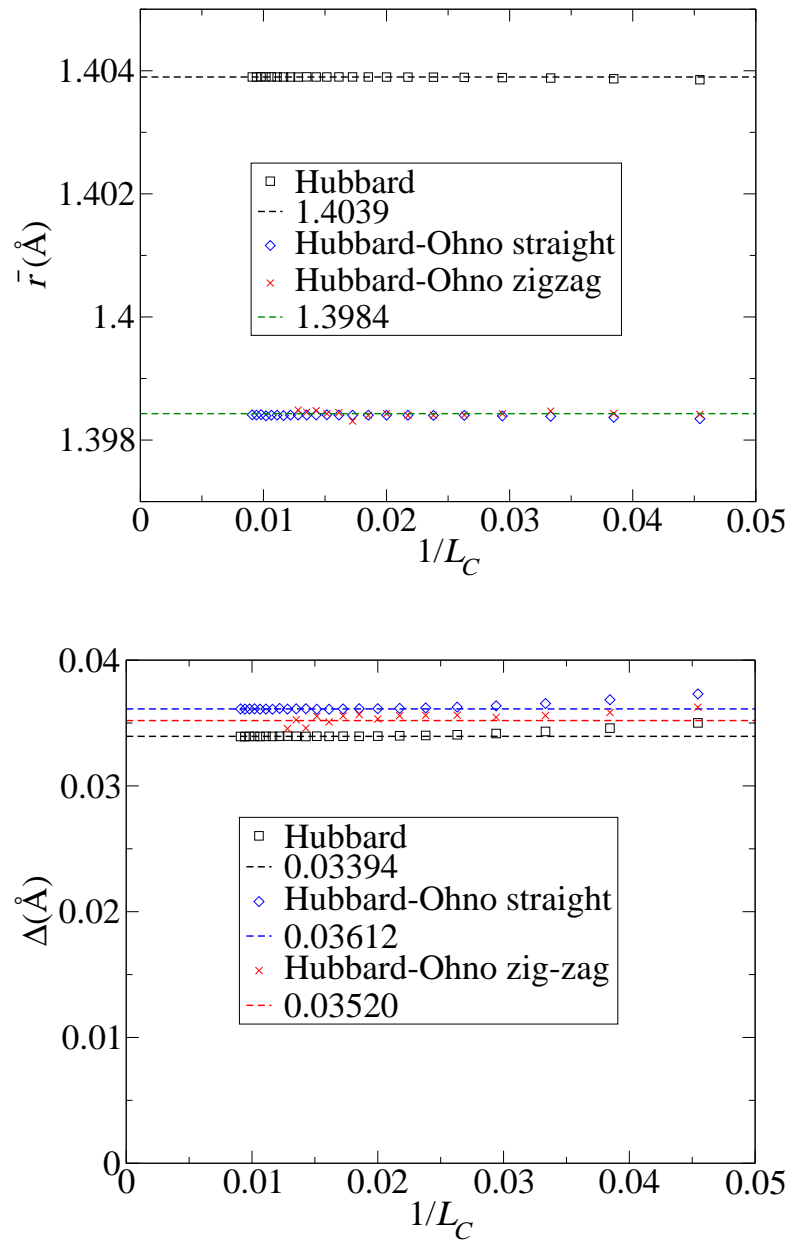

FIG. 12. Average bond length and dimerization for the Hückel-Hubbard(-Ohno) model with $t_{0}=2.5 \mathrm{eV}, U=6 \mathrm{eV}$ $\left[V=3 \mathrm{eV}, \epsilon_{d}=2.3\right], \alpha=4.0 \mathrm{eV} / \AA, K_{\sigma, 0}=-4.8 \mathrm{eV} / \AA$, $K_{\sigma, 1}=42 \mathrm{eV} / \AA^{2}$, and $\tilde{C}_{b}=3.5 \mathrm{eV} / \mathrm{rad}^{2}$ as a function of the inverse chain length for open boundary conditions (obc, DMRG, $L_{C}=2 L+2$ ). For the constant extrapolation of the DMRG data, system sizes $L_{C} \geq 50$ are used.

As seen from Fig. 12, the influence of the Coulomb interaction on the average bond length is very small. The Hubbard interaction increases the average bond length by only $0.015 \AA$. The long-range Ohno interaction reduces the average bond length again by a small amount so that the increase in bond length is below $0.01 \AA$ from the Hückel to the Hückel-Hubbard-Ohno model. Fig. 12 also shows that the zig-zag geometry has a negligible influence on the average bond length.

In contrast, the Coulomb interaction is very important for the size of the dimerization. The dimerization for the Hückel-Hubbard(-Ohno) model with $U=6 \mathrm{eV}$ (and $V=3 \mathrm{eV}, \epsilon_{d}=2.3$ ), is larger than that for the bare Hückel model by a factor of two, from $\Delta^{\mathrm{H}} \approx 0.02 \AA$ to $\Delta^{\mathrm{HH}} \approx 0.04 \AA$, the experimental value for transpolyacetylene. As seen from Fig. 12 the main reason for this large increase is the Hubbard interaction whereas the Ohno contribution is fairly small, below $0.002 \AA$. For this reason, the chain geometry does not play a big role. As seen from the figure, the difference between the dimerization for straight and zig-zag chains is at most $0.001 \AA$.

Finite-size effects are negligibly small. The average bond length is essentially independent of $L_{C}$, and the dimerization becomes independent of system size for $L_{C} \gtrsim 50$. Therefore, we fit the DMRG data to a constant in Fig. 12 .

\section{B. Optical phonons}

\section{Linear chain}

In Fig. 13 we show the phonon frequency as a function of inverse system size for the Hückel-Hubbard and Hückel-Hubbard-Ohno models. For comparison we note that the bare optical frequency for a chain without electron-phonon coupling $(\alpha=0)$ is given by $\omega_{0}=$ $\sqrt{4 K_{\sigma, 1} / M}=1951 \mathrm{~cm}^{-1} \cdot 20$

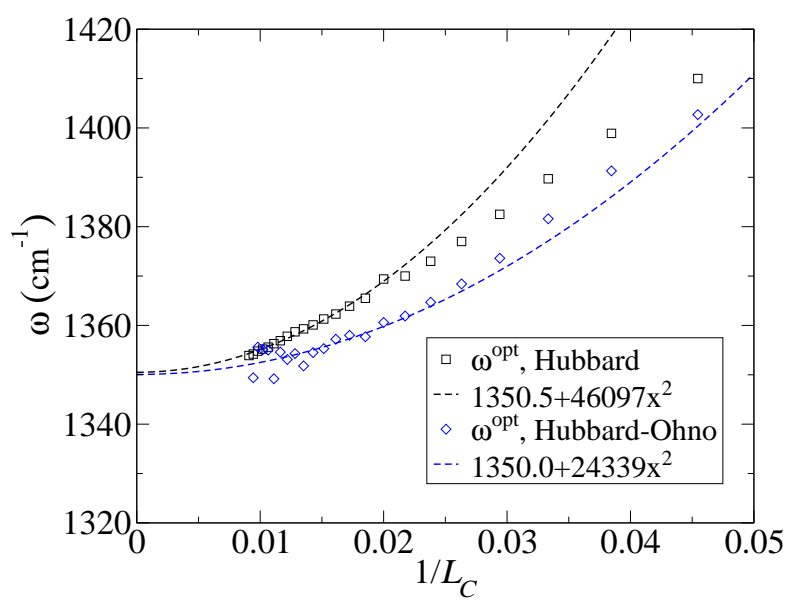

FIG. 13. Phonon frequency for the Hückel-Hubbard(-Ohno) model on a straight chain as a function of inverse chain length for the same parameter set as in Fig. 12 For the quadratic extrapolation of the DMRG data, system sizes $L_{C} \geq 50$ are used.

For our parameter set, the Coulomb interaction leads to a single-particle gap of several eV. As seen from Fig. 13, the phonon frequency shows a very moderate finite-size dependence and can nicely be extrapolated to the thermodynamic limit. Moreover, the long-range Ohno interaction shifts the phonon frequency by only $10 \mathrm{~cm}^{-1}$, or less, i.e., the frequency shift is almost negligibly small, below $0.1 \%$. This observation is helpful for parameter optimizations because DMRG calculations for 
the Hückel-Hubbard model are much less time consuming than those for the Hückel-Hubbard-Ohno model due to the absence of long range Coulomb interaction. It was already noted in Ref. 35] that the long-range part of the Coulomb interaction barely influences the effective $\sigma$-bond spring constant.

\section{Zig-zag chain}

Lastly, we present results for the zig-zag chain with an angle $\Theta_{0}=120^{\circ}$ between adjacent double and single bonds. In Fig. 14 we show the frequencies of the optical phonons for the Hückel-Hubbard-Ohno model. As also seen for the straight chain in Fig. 13, the phonon frequencies in the $A_{g}$ symmetry sector are shifted upward by the Coulomb interaction. As for the straight chain, the long-range Coulomb interaction does not affect the phonon frequencies significantly. The changes are again of the order of $10 \mathrm{~cm}^{-1}$ or one percent.

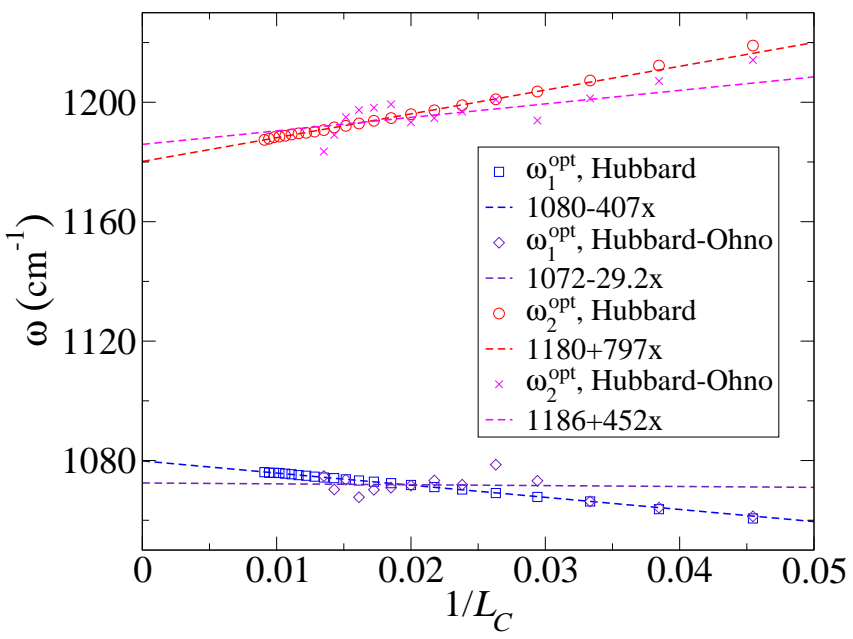

FIG. 14. Phonon frequencies for the Hückel-Hubbard(-Ohno) model on a zig-zag chain as a function of inverse chain length for the same parameter set as in Fig. 12. For the linear extrapolation of the DMRG data, system sizes $L_{C} \geq 30$ are used.

The optical phonons are split in energy by about $100 \mathrm{~cm}^{-1}$ for all chain lengths so that an anti-crossing of the phonons as a function of frequency is not observed, in contrast to the bare Hückel model with a small gap. The system sizes $L_{C} \approx 100$ are still too small to permit a quadratic fit of the phonon frequencies as a function of system size. We expect, however, that the linear frequency extrapolations lead to the correct phonon frequencies in the thermodynamic limit, to within $10 \mathrm{~cm}^{-1}$ or one percent.

For completeness, in Fig. 15 we show the entries of the dynamical matrix $\tilde{K}_{i, j}$ in the $A_{g}$-sector as a function of system size for the Hückel model with a small gap, and the Hückel-Hubbard(-Ohno) model. In the Hückel model
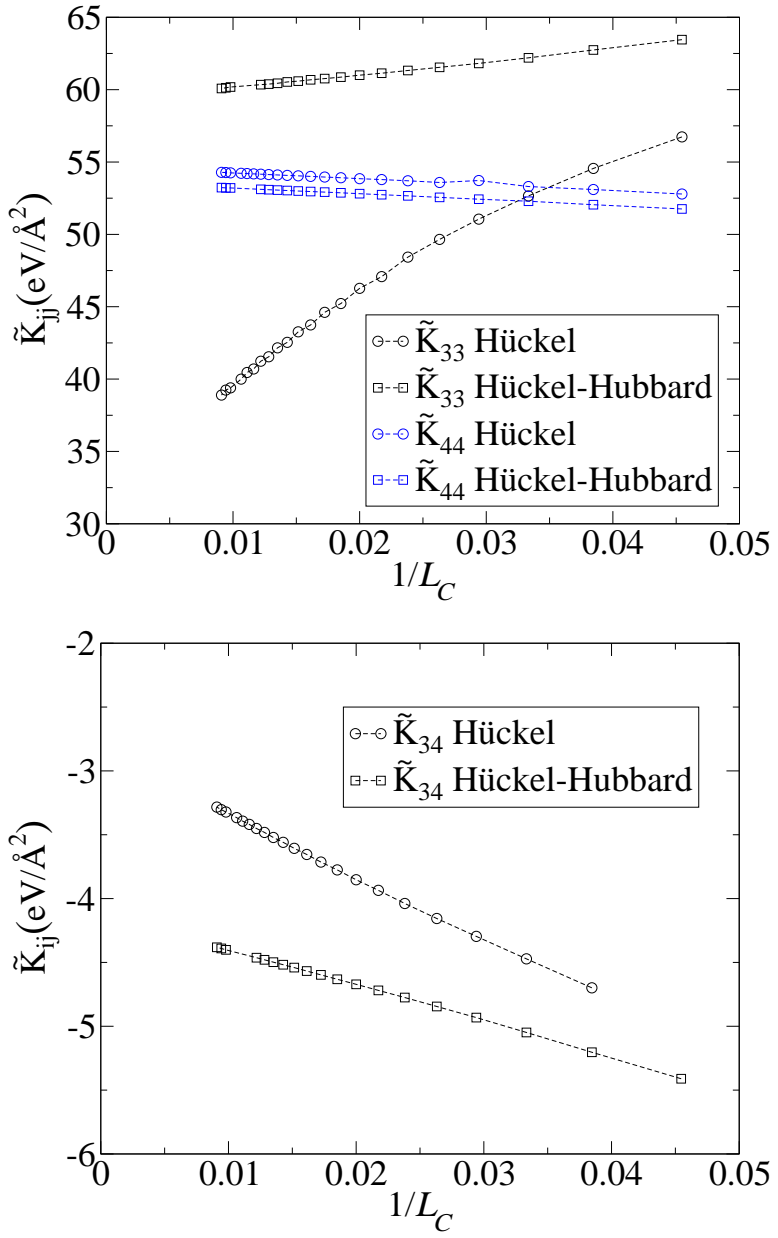

FIG. 15. Entries of the dynamical matrix $\tilde{K}_{i, j}$ in the $A_{g}$ symmetry sector for the Hückel-Hubbard(-Ohno) model with $t_{0}=$ $2.5 \mathrm{eV}, \alpha=4.0 \mathrm{eV} / \AA, K_{\sigma, 0}=-4.8 \mathrm{eV} / \AA, K_{\sigma, 1}=42 \mathrm{eV} / \AA^{2}$, $\tilde{C}_{b}=3.5 \mathrm{eV} / \mathrm{rad}^{2}$, [and $U=6 \mathrm{eV}\left(V=3 \mathrm{eV}, \epsilon_{d}=2.3\right)$ ], as a function of the inverse chain length for open boundary conditions (obc, DMRG, $L_{C}=2 L+2$ ).

with a small gap, the matrix element $\tilde{K}_{33}$ for anti-phase distortions in the $x$-direction displays a large finite-size dependence, similar to the finite-size gap in Fig. 9. For this reason, $\tilde{K}_{33}$ equals the value for $\tilde{K}_{44}$ around $L_{C}=30$ which leads to the avoided crossing of the phonon frequencies seen in Fig. 11]. This drastic finite-size behavior is suppressed by the Hubbard(-Ohno) interaction. The Coulomb repulsion leads to a much larger single-particle gap that is well converged as a function of inverse system size for $L_{C} \gtrsim 50$. As a consequence, the phonon frequencies shown in Fig. 14 are well separated and do not show signatures of an avoided crossing. Note that $\tilde{K}_{44}$ and $\tilde{K}_{34}=\tilde{K}_{43}$ are quite similar, i.e., the Coulomb interaction plays a minor role for distortions that involve the $y$ direction, perpendicular to the chain orientation. This is not surprising because in our quasi one-dimensional system the electron wave functions are extended only along the $x$-direction. 


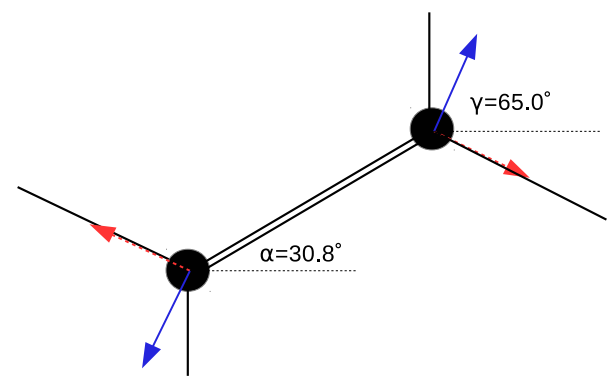

FIG. 16. Optical phonon distortions associated with the antiphase stretching mode at frequency $\omega_{2}^{\text {opt }}$ (red dotted arrows) and with the anti-phase swinging mode at frequency $\omega_{1}^{\text {opt }}$ (blue arrows) for the Hückel-Hubbard model on $L_{C}=66$ sites for the same parameter set as in Fig. 12

In Fig. 16 we show the eigenvectors for the anti-phase oscillations for the Hückel-Hubbard model for $U=6 \mathrm{eV}$ and $L_{C}=66$. The eigenvectors for the lattice distortions for the Hückel-Hubbard(-Ohno) model are still given by eq. (33) but now we have $\gamma_{\mathrm{HH}}=1.13 \mathrm{rad}=65^{\circ}$. The eigenvectors result from the diagonalization of the dynamical matrix with the entries $\tilde{K}_{33}=60.59, \tilde{K}_{34}=$ $\tilde{K}_{43}=-4.54$, and $\tilde{K}_{44}=53.00$ (in units of $\mathrm{eV} / \AA^{2}$ ).

A comparison of Fig. 8 for the Hückel model with a large gap and of Fig. 16 for the Hückel-Hubbard model shows that the eigenvector pairs are rotated against each other by about 45 degrees. In the Hückel-Hubbard model, there is no clear distinction between a 'stretching' and a 'swinging' mode. Instead, the phonon mode with the (higher) energy $\omega_{2}^{\text {opt }}$ corresponds to a stretching of the single bond whereas the phonon mode with the (lower) energy $\omega_{1}^{\text {opt }}$ involves a stretching (and swinging) of the double bond. Therefore, both modes are very similar in their finite-size behavior.

\section{SUMMARY AND CONCLUSIONS}

In this work, we calculated the optical phonon frequencies for the Hückel and Hückel-Hubbard(-Ohno) models on linear and zig-zag chains with up to $L_{C}=110$ sites using the Density-Matrix Renormalization-Group (DMRG) method. When the electron-electron interaction is absent (Hückel model), the (optical) phonon spectrum can be calculated analytically for periodic boundary conditions and thus provides a benchmark test for the DMRG calculations. For systems with a large single-particle gap, the analytic and numerical results for the average bond length, the dimerization, the single-particle gap, and the optical phonon frequencies agree very well, which validates the applicability of the DMRG approach.

When the Peierls gap is small, the Kohn anomaly leads to an avoided crossing of the two optical phonon branches of the zig-zag chain as a function of inverse system size. Numerically, it is difficult to reach system sizes where the frequency of the bond-stretching phonon becomes close to its value in the thermodynamic limit. Moreover, we treated the lattice deformations classically. This is justified in presence of a large single-particle gap but causes problems when the system is close to the Peierls transition. In systems with a small Peierls gap, the phonons ought to be treated quantum mechanically. 12

In presence of a sizable Hubbard interaction, of the order of half the bandwidth, the gap for single-particle excitations is large and the Kohn anomaly is suppressed. Finite-size effects of the average bond length, the dimerization, the single-particle gap, and the optical phonon frequencies are small, and the DMRG results can be extrapolated reliably to the thermodynamic limit. For the zig-zag chain we find that the two optical phonon modes are energetically well separated for all system sizes. We find that a moderate Ohno repulsion increases the singleparticle gap whereas it barely influences the average bond length, the dimerization, and the optical phonon frequencies. When we increase the interaction parameters we observe that a larger Hubbard- $U$ and/or a larger Ohno- $V$ increase both the band gap and the dimerization substantially. Contrary to this, the average bond length remains insensitive to the Coulomb interaction.

The Hückel-Hubbard-Ohno model on a zig-zag chain includes the basic structural elements and fundamental electronic components for the description of optical phonons in trans-polyacetylene. Raman spectroscopy reveals four $A_{g}$ phonon modes ${ }^{27-30}$ of which two display a pronounced dependence on the chain length. ${ }^{27}$ Their frequencies are $\omega_{\mathrm{A}} \approx 1070 \mathrm{~cm}^{-1}$ and $\omega_{\mathrm{C}} \approx 1460 \mathrm{~cm}^{-1}$. Apparently, $\omega_{A}$ is close to $\omega_{1}^{\text {opt }} \approx 1080 \mathrm{~cm}^{-1}$, as derived for the Hückel-Hubbard-Ohno model. Even $\omega_{C}$ is in the range of $\omega_{2}^{\text {opt }} \approx 1180 \mathrm{~cm}^{-1}$. Note, however, that the modes $\mathrm{A}$ and $\mathrm{C}$ seen in experiment couple to the motion of the hydrogen atoms whereas we studied solely the motion of the carbon atoms. Therefore, a realistic description of optical phonons requires the inclusion of the hydrogen atoms. Work in this direction is in progress.

\section{Appendix A: Non-interacting electrons}

In this appendix, we provide analytical expressions for the ground-state conformation and optical phonons for non-interacting electrons on straight and zig-zag chains with periodic boundary conditions.

\section{Ground-state energy and bond lengths}

The operator for the kinetic energy reads

$$
\begin{aligned}
\hat{T}= & -\sum_{\sigma} \sum_{n=1}^{L} t_{d}\left(\hat{c}_{2 n-1, \sigma}^{+} \hat{c}_{2 n, \sigma}+\hat{c}_{2 n, \sigma}^{+} \hat{c}_{2 n-1, \sigma}\right) \\
& -\sum_{\sigma} \sum_{n=1}^{L} t_{s}\left(\hat{c}_{2 n, \sigma}^{+} \hat{c}_{2 n+1, \sigma}+\hat{c}_{2 n+1, \sigma}^{+} \hat{c}_{2 n, \sigma}\right)
\end{aligned}
$$


where

$$
\begin{aligned}
& t_{d}=t_{0} \exp \left(-\left(r_{d}-r_{0}\right) \alpha / t_{0}\right) \\
& t_{s}=t_{0} \exp \left(-\left(r_{s}-r_{0}\right) \alpha / t_{0}\right)
\end{aligned}
$$

are the electron transfer matrix elements for short bonds (atomic distance $r_{d}$ ) and long bonds (atomic distance $r_{s}$ ). The length of the bonds is modulated periodically due to the Peierls effect.

The Hamiltonian is readily diagonalized in momentum space, 20

$$
\begin{aligned}
\hat{T} & =\sum_{k, \sigma} E(k)\left(\hat{a}_{k, \sigma,+}^{\dagger} \hat{a}_{k, \sigma,+}-\hat{a}_{k, \sigma,-}^{\dagger} \hat{a}_{k, \sigma,-}\right), \\
E\left(k_{m}\right) & =\sqrt{\left(t_{s}+t_{d}\right)^{2} \cos ^{2}\left(k_{m}\right)+\left(t_{s}-t_{d}\right)^{2} \sin ^{2}\left(k_{m}\right)}, \\
k_{m} & =(\pi / L) m, m=-(L / 2)+1, \ldots,(L / 2) .
\end{aligned}
$$

The kinetic energy per unit cell as a function of $r_{s}$ and $r_{d}$ is given by

$$
T\left(r_{s}, r_{d}\right)=-\frac{2}{L} \sum_{m=-L / 2+1}^{L / 2} E\left(k_{m}\right) .
$$

The parameters $r_{d}$ and $r_{s}$ follow from the minimization of the kinetic energy and the potential energy per unit cell.

The potential energy is given by the compression energy per unit cell,

$$
\begin{aligned}
E_{\mathrm{CC}}\left(r_{s}, r_{d}\right) & =V_{\sigma}\left(r_{s}\right)+V_{\sigma}\left(r_{d}\right), \\
V_{\sigma}(r) & =K_{\sigma, 0}\left(r-r_{0}\right)+\frac{K_{\sigma, 1}}{2}\left(r-r_{0}\right)^{2},
\end{aligned}
$$

see eq. (11). The optimal values $\left(R_{s}, R_{d}\right)$ for the bond lengths follow from the (numerical) solution of the equations

$$
\begin{aligned}
V_{\sigma}^{\prime}\left(R_{s}\right) & =\left.\frac{\partial T\left(r_{s}, r_{d}\right)}{\partial r_{s}}\right|_{r_{s}=R_{s}, r_{d}=R_{d}}, \\
V_{\sigma}^{\prime}\left(R_{d}\right) & =\left.\frac{\partial T\left(r_{s}, r_{d}\right)}{\partial r_{d}}\right|_{r_{s}=R_{s}, r_{d}=R_{d}} .
\end{aligned}
$$

Note that $\left(R_{s}, R_{d}\right)$ depend on the number of unit cells $L$ of the chain.

We rewrite the total energy in terms of the average bond length and the dimerization using the dimensionless variables

$$
\begin{aligned}
& s=\frac{\alpha\left(r_{d}+r_{s}-2 r_{0}\right)}{2 t_{0}}, \\
& v=\frac{\alpha\left(r_{s}-r_{d}\right)}{2 t_{0}} .
\end{aligned}
$$

Then, the total energy per unit cell becomes

$$
\begin{aligned}
E / L= & -4 t_{0} e^{-s} \cosh (v) \\
& \times \frac{1}{L} \sum_{|k| \leq \pi / 2} \sqrt{\cos ^{2}(k)+\tanh ^{2}(v) \sin ^{2}(k)} \\
& +\frac{2 K_{\sigma, 0} t_{0}}{\alpha} s+\frac{K_{\sigma, 1} t_{0}^{2}}{\alpha^{2}}\left(s^{2}+v^{2}\right) .
\end{aligned}
$$

At the optimal values $s_{0}$ and $v_{0}$ the gradient of the energy vanishes. This leads to the coupled equations

$$
\begin{aligned}
0= & \frac{2 K_{\sigma, 0} t_{0}}{\alpha}+\frac{2 K_{\sigma, 1} t_{0}^{2}}{\alpha^{2}} s_{0} \\
& +4 t_{0} e^{-s_{0}} \cosh \left(v_{0}\right) \\
& \times \frac{1}{L} \sum_{|k| \leq \pi / 2} \sqrt{\cos ^{2}(k)+\tanh ^{2}\left(v_{0}\right) \sin ^{2}(k)} \\
0= & \frac{2 K_{\sigma, 1} t_{0}^{2}}{\alpha^{2}} v_{0} \\
& -4 t_{0} e^{-s_{0}} \frac{1}{L} \sum_{|k| \leq \pi / 2} \frac{\sinh \left(2 v_{0}\right)}{\sqrt{2 \cos (2 k)+2 \cosh \left(2 v_{0}\right)}},
\end{aligned}
$$

which thus define $R_{s}$ and $R_{d}$ from eq. (A7). For noninteracting electrons, the bond lengths are independent of the bond angles so that the above expressions apply for both straight and zig-zag chains. The expressions are evaluated using MATHEMATICA. 36

\section{Optical phonons}

a. Straight chain

For an optical phonon, the displacements leave the length of the unit cell invariant, $r_{d}+r_{s}=R_{d}+R_{s}=a_{0}$. The optical phonon corresponds to an anti-phase oscillation of the atoms in the unit cell. Its effective spring constant is given by

$$
K_{\mathrm{eff}}=\left.\frac{\alpha^{2}}{2 t_{0}^{2}} \frac{\partial^{2}(E / L)}{\partial v^{2}}\right|_{s=s_{0}, v=v_{0}}
$$

where we included the Jacobi determinant of the transformation (A7). Explicitly,

$$
\begin{aligned}
K_{\text {eff }} & =K_{\sigma, 1}-\frac{\alpha^{2}}{t_{0}} e^{-s_{0}} \frac{1}{L} \sum_{|k| \leq \pi / 2} H(k) \\
H(k) & =\frac{3+4 \cos (2 k) \cosh \left(2 v_{0}\right)+\cosh \left(4 v_{0}\right)}{\sqrt{2}\left(\cos (2 k)+\cosh \left(2 v_{0}\right)\right)^{3 / 2}},
\end{aligned}
$$

and

$$
\omega^{\mathrm{opt}}=\sqrt{\frac{4 K_{\mathrm{eff}}}{M}} .
$$

For $t_{0}=2.5 \mathrm{eV}, \alpha=4.0 \mathrm{eV} / \AA K_{\sigma, 0}=-4.8 \mathrm{eV} / \AA$, $K_{\sigma, 1}=42 \mathrm{eV} / \AA^{2}$, and $M=12 u$ we find $\omega^{\text {opt }}=$ $864.5 \mathrm{~cm}^{-1}$ for an infinitely long chain. For finite chains, the results are shown in Fig. 10. We compare these results with a bare straight chain without itinerant electrons for which $\omega^{\text {opt,bare }}=\omega_{0}=\sqrt{4 K_{\sigma, 1} / M}=$ $1951.2 \mathrm{~cm}^{-1}$. Apparently, the renormalization of the phonon frequency is quite large, about a factor of two, $\omega^{\mathrm{opt}} / \omega_{0}=0.443$. 


\section{b. Zig-zag chain}

For the zig-zag chain, the size of the unit cell is given by

$$
a_{0}=\sqrt{R_{s}^{2}+R_{d}^{2}+R_{s} R_{d}}
$$

because all bonds form an angle of $\Theta_{0}=2 \pi / 3$. Then, the carbon atoms are located at $(n=1, \ldots, L)$

$$
\begin{aligned}
X_{2 n-1} & =(n-1) a_{0}, \\
Y_{2 n-1} & =0, \\
X_{2 n} & =(n-1) a_{0}+R_{d} \sqrt{1-\frac{3 R_{s}^{2}}{4 a_{0}^{2}}}, \\
Y_{2 n} & =\frac{\sqrt{3} R_{s} R_{d}}{2 a_{0}} .
\end{aligned}
$$

Since $\left(R_{s}, R_{d}\right)$ are determined from eq. (A6), the groundstate conformation is fixed for a given number of unit cells $L$.

Optical phonons induce displacements of the four atoms in the unit cell in the directions $x$ and $y$. The coordinates in the presence of the four displacements $\vec{\delta}=\left(\delta_{1}, \delta_{2}, \delta_{3}, \delta_{4}\right)$ are defined by

$$
\begin{aligned}
x_{2 n-1} & =X_{2 n-1}+\delta_{3}, \\
y_{2 n-1} & =Y_{2 n-1}+\delta_{4}, \\
x_{2 n} & =X_{2 n}+\delta_{1}, \\
y_{2 n} & =Y_{2 n}+\delta_{2} .
\end{aligned}
$$

The distortions $\vec{\delta}$ result in a change of the bond lengths. The distances between neighboring atoms are given by

$$
\begin{aligned}
& r_{s}(\vec{\delta})=\sqrt{\left(x_{2 n-1}+a_{0}-x_{2 n}\right)^{2}+\left(y_{2 n-1}-y_{2 n}\right)^{2}}, \\
& r_{d}(\vec{\delta})=\sqrt{\left(x_{2 n}-x_{2 n-1}\right)^{2}+\left(y_{2 n}-y_{2 n-1}\right)^{2}}
\end{aligned}
$$

for all $1 \leq n \leq L$, and we may set $n=1$ for convenience.

The kinetic energy per unit is still given by eq. (A4),

$$
T\left(r_{s}(\vec{\delta}), r_{d}(\vec{\delta})\right)=-\frac{2}{L} \sum_{m=-L / 2+1}^{L / 2} E\left(k_{m}\right),
$$

where $E\left(k_{m}\right)$ depends on $\vec{\delta}$ via the tunnel amplitudes $t_{s, d}\left(r_{s}(\vec{\delta}), r_{d}(\vec{\delta})\right)$, see eqs. A2 , A3 ). The bond-length distortions result in the potential-energy contributions

$$
E_{\mathrm{CC}}(\vec{\delta})=V_{\sigma}\left(r_{s}(\vec{\delta})\right)+V_{\sigma}\left(r_{d}(\vec{\delta})\right)
$$

and

$$
E_{\mathrm{CCb}}(\vec{\delta})=C_{\mathrm{b}}\left[\cos (\vartheta(\vec{\delta}))-\cos \left(\Theta_{0}\right)\right]^{2}
$$

per unit cell with

$$
\begin{aligned}
-\cos (\vartheta(\vec{\delta}))= & \frac{\left(a_{0}-X_{2}+\delta_{3}-\delta_{1}\right)\left(X_{2}+\delta_{1}-\delta_{3}\right)}{r_{s}(\vec{\delta}) r_{d}(\vec{\delta})} \\
& +\frac{\left(\delta_{4}-Y_{2}-\delta_{2}\right)\left(Y_{2}+\delta_{2}-\delta_{4}\right)}{r_{s}(\vec{\delta}) r_{d}(\vec{\delta})} .(\mathrm{A} 20)
\end{aligned}
$$

The total energy per unit cell becomes

$$
E_{\text {struc }}(\vec{\delta})=T\left(r_{s}(\vec{\delta}), r_{d}(\vec{\delta})\right)+E_{\mathrm{CC}}(\vec{\delta})+E_{\mathrm{CC} \mathrm{b}}(\vec{\delta}) .
$$

By construction, see eq. A6 , the gradient of $E_{\text {struc }}(\vec{\delta})$ vanishes at $\vec{\delta}=\overrightarrow{0}$, as it must be for a stable ground-state conformation.

The entries of the dynamical matrix $\underline{\underline{K}}$ are determined from

$$
K_{i, j}=\left.\frac{\partial^{2} E_{\mathrm{struc}}(\vec{\delta})}{\partial \delta_{i} \partial \delta_{j}}\right|_{\vec{\delta}=\overrightarrow{0}} .
$$

The dynamical matrix is given in units of $\mathrm{eV} / \AA^{2}$. As seen from eqs. (A16) and (A20), only the combinations $\delta_{1}-\delta_{3}$ and $\delta_{2}-\delta_{4}$ appear in the energy. Therefore, two of the eigenvalues of the dynamical matrix are zero, as required for optical phonons. The two zero eigenvalues correspond to the motion of the chain as a whole into the $x$-direction [displacement eigenvector $\vec{\delta}_{x}=(1,0,1,0)$ ] and in the $y$ direction [displacement eigenvector $\vec{\delta}_{y}=(0,1,0,1)$ ]. We verify numerically that the other two eigenvalues are positive, as it must be for a stable ground-state configuration.

\section{Appendix B: Dynamical matrix from DMRG calculations}

In this appendix, we provide some details of our DMRG algorithm, we discuss how the ground-state conformation is obtained iteratively, and we show how to calculate the elements of the dynamical matrix straightforwardly using DMRG.

\section{DMRG algorithm}

We investigate the Hückel, the Hückel-Hubbard, and the Hückel-Hubbard-Ohno models with open boundary condition applying the density-matrix renormalization group (DMRG) method ${ }^{22,23}$ We perform simulations on system sizes from $L_{\mathrm{C}}=10$ up to $L_{\mathrm{C}}=110\left(L_{\mathrm{C}}=2 L+2\right)$ in steps $\Delta L_{\mathrm{C}}=4$. The precision of the calculations is controlled in terms of the dynamic block-state selection (DBSS) approach, $, 37,38$ whereby we keep up to 1000 block states and perform six sweeps.

Using the DMRG as a kernel, we implement a selfconsistent geometrical optimization method in order to obtain the relaxed geometry, i.e., the geometry with the lowest ground-state energy. In each iteration step the DMRG solves the electronic Hamiltonian problem $\hat{H}_{\mathrm{el}}$ (8) for a fixed atomic conformation. Moreover, the algorithm provides the transition and occupation probabilities in (2) and (6). Using these expectation values for the construction of the electronic energy term (8), the total energy (16) becomes a function of the atom coordinates $\left(\left\{x_{n}\right\},\left\{y_{n}\right\}\right)$ that is minimized using a gradient search. 


\section{Optimization of the ground-state structure}

The minimization of the total energy of the structure, eq. (16), is achieved iteratively. For a fast convergence, an educated guess for the ground state conformation is helpful. We start with the homogeneous conformation investigating small system size with $L=2$ unit cells. We set $r_{\mathrm{s}, l}=r_{\mathrm{d}, l}=r_{0}=1.4 \AA$ in eq. A14 so that $a_{0}=\sqrt{3} r_{0}$ is the bare size of the unit cell. In order to speed up convergence, for larger systems with $L>2$ unit cells a distorted initial geometry is constructed utilizing the optimized geometry of system with $L-2$ unit cells. We set $r_{\mathrm{s}, l}=r_{\mathrm{d}, l}=r_{0}=1.4 \AA$ in eq. A14 so that $a_{0}=\sqrt{3} r_{0}$ is the bare size of the unit cell.

\section{a. Iteration}

The algorithm seeks for the self-consistent solution of the structural and the electronic problem:

1. The structure determines the parameters for the $\pi$ electrons' nearest-neighbor transfer and their mutual Coulomb interaction;

2. The potential energy landscape shaped by the $\sigma$ bonds and the $\pi$-electrons defines the structure.

Correspondingly, the algorithm proceeds as follows.

1. We define for the $k$ th iteration $\left(k=1,2, \ldots, k_{\max }\right)$ for $n=1,2, \ldots, 2 L$

$$
\vec{r}_{n}^{(k)}=\left(\begin{array}{c}
x_{n}^{(k)} \\
y_{n}^{(k)}
\end{array}\right)
$$

Typically, $k_{\max }=10$ is sufficient to obtain convergence.

2. The $k$ th DMRG run is based on the atomic positions $\vec{r}_{n}^{(k-1)}\left(k=1,2, \ldots, k_{\max }\right)$. It provides the elements of the single-particle density matrix for nearest neighbors $(n=1,2, \ldots, 2 L-1)$

$$
P_{n, \sigma}^{(k)} \equiv\left\langle\hat{c}_{n, \sigma}^{\dagger} \hat{c}_{n+1, \sigma}+\hat{c}_{n+1, \sigma}^{\dagger} \hat{c}_{n, \sigma}\right\rangle_{0}^{(k)}
$$

the local double occupancy,

$$
D_{n}^{(k)} \equiv\left\langle\left(\hat{n}_{n, \uparrow}-1 / 2\right)\left(\hat{n}_{n, \downarrow}-1 / 2\right)\right\rangle_{0}^{(k)},
$$

and the elements for the density-density correlation function $(n, j=1, \ldots, 2 L, n \neq j)$

$$
C_{n j}^{(k)} \equiv\left\langle\left(\hat{n}_{n}-1\right)\left(\hat{n}_{j}-1\right)\right\rangle_{0}^{(k)}
$$

The positions $\vec{r}_{n}^{(k-1)}$ and the matrix elements $P_{n, \sigma}^{(k)}$, $D_{n}^{(k)}$, and $C_{n j}^{(k)}$ determine the total energy of the structure $E_{\text {struc }}^{(k)}$, see eq. (16).
3. The iteration cycle stops if $k=k_{\max }$ is reached or if, for $k \geq 2$,

$$
\frac{\left|E_{\text {struc }}^{(k-1)}-E_{\text {struc }}^{(k)}\right|}{2 L+2}<\epsilon
$$

with $\epsilon=10^{-4}$ for a sufficient accuracy.

If neither of the two conditions is fulfilled, we determine new atomic positions. Starting from the configuration $\vec{r}_{n}^{(k-1)}$ the energy functional $E_{\text {struc }}$ for fixed $P_{n, \sigma}^{(k)}, D_{n}^{(k)}$, and $C_{n j}^{(k)}$ is minimized with respect to the atomic positions $\vec{r}_{n}$. A conjugate gradient method requires the derivatives of $E_{\text {struc }}$ with respect to the atomic positions. The gradients are calculated analytically using the HellmanFeynman theorem ${ }^{39-41}$ The corresponding expressions are collected in the supplemental material.

After convergence we find the new positions $\vec{r}^{(k)}$ and the energetic minimum defines $E_{\text {struc }}^{(k)}$.

The steps 2 and 3 are iterated until the iteration cycle stops.

\section{Calculation of the dynamical matrix}

\section{a. Diagonal terms}

We start with the diagonal terms and consider a small distortion with amplitude $\Delta_{i}$ in the $i$ th component of $\vec{p}_{l}$

$$
e\left(\Delta_{i}, L\right) \equiv \frac{E_{\text {struc }}\left(\left\{\vec{R}_{l}+\Delta_{i} \vec{e}_{i}\right\}\right)-E_{0}}{L}=\frac{1}{2} K_{i, i} \Delta_{i}^{2},
$$

up to second order in $\Delta_{i}$. Thus, we have

$$
K_{i, i}(L)=\lim _{\Delta_{i} \rightarrow 0} \frac{2 e\left(\Delta_{i}\right)}{\Delta_{i}^{2}} .
$$

In our approach, we calculate $e\left(\Delta_{i}, L\right)$ for chains with up to $L_{C}=110$ sites and for three values $\Delta_{i}^{(k)}$. Then, we apply a quadratic fit of the parabola

$$
\varepsilon(\Delta, L)=\left(K_{i, i}(L) / 2\right) \Delta^{2}
$$

to the three points $\left(\Delta^{(k)}, e\left(\Delta^{(k)}, L\right)\right)$ and the origin. The curvature defines $K_{i, i}$.

We set

$$
\Delta^{(1)}=0.005 \AA, \Delta^{(2)}=0.010 \AA, \Delta^{(3)}=0.015 \AA .
$$

The choice of these values is derived from typical values for the displacements. For a phonon of energy $\hbar \omega$, the average square displacement at thermal energy $k_{\mathrm{B}} T$ is $\left\langle x^{2}\right\rangle=k_{\mathrm{B}} T /\left(M \omega^{2}\right)$. For a typical optical phonon energy of $\hbar \omega=0.2 \mathrm{eV}$ in polyacetylene $\left(1 / \lambda=1600 \mathrm{~cm}^{-1}\right)$, the square average displacement for carbon $(M=12 u, 1 u=$ $\left.0.9315 \mathrm{GeV} / c^{2}\right)$ at room temperature $\left(k_{\mathrm{B}} T=0.025 \mathrm{eV}\right)$ 


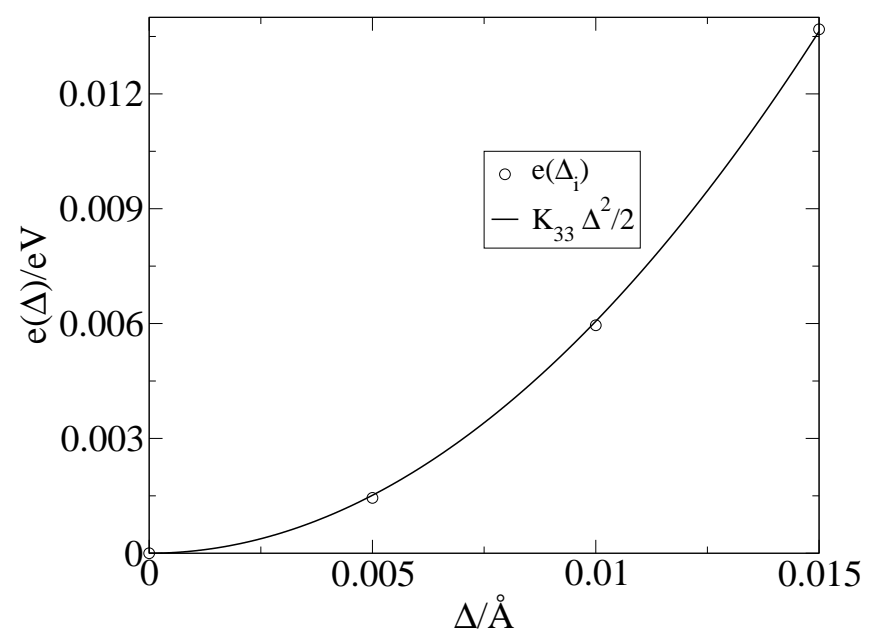

FIG. 17. Excitation energy for distortions of the first carbon atom in each unit cell in the $x$-direction for the HückelHubbard model on a zig-zag chain with $t_{0}=2.5 \mathrm{eV}, \alpha=$ $4.0 \mathrm{eV} / \AA, U=6 \mathrm{eV}, K_{\sigma, 0}=-4.8 \mathrm{eV} / \AA$, and $K_{\sigma, 1}=$ $42 \mathrm{eV} / \AA^{2}$ in units of $\mathrm{eV}$ as a function of the distortion $\Delta$ in units of $\AA$ for open boundary conditions. The straight line is a parabolic fit with $K_{33}=60.59 \mathrm{eV} / \AA^{2}$.

is $\sqrt{\left\langle x_{\mathrm{C}}^{2}\right\rangle} \approx 0.015 \AA$; for the calculation we used $\hbar c=$ $1974 \mathrm{eVA}$.

As an example, in Fig. 17 we show the extrapolation of $\tilde{K}_{33}$ for the Hückel-Hubbard model for $U=6 \mathrm{eV}$ and $L=32$ unit cells on a zig-zag chain. It is seen that the extrapolation is stable and provides a reliable value for the 'spring constant' $\tilde{K}_{33}=60.69 \mathrm{eV} / \AA^{2}$. Likewise, we obtain $\tilde{K}_{44}=53.00 \mathrm{eV} / \AA^{2}$.

\section{b. Off-diagonal terms}

For the off-diagonal terms, $1 \leq i<j \leq 4$, we consider

$$
\begin{aligned}
e_{2}\left(\Delta_{i}, \Delta_{j}, L\right) \equiv & E_{\text {struc }}\left(\left\{\vec{R}_{l}+\Delta_{i} \vec{e}_{i}+\Delta_{j} \vec{e}_{j}\right\}\right) / L \\
& -E_{\text {struc }}\left(\left\{\vec{R}_{l}+\Delta_{i} \vec{e}_{i}\right\}\right) / L \\
& -E_{\text {struc }}\left(\left\{\vec{R}_{l}+\Delta_{j} \vec{e}_{j}\right\}\right) / L+E_{0} / L \\
= & K_{i, j} \Delta_{i} \Delta_{j}+\mathcal{O}\left(\Delta^{3}\right) .
\end{aligned}
$$

We fix $\Delta_{i}$ at its smallest value, $\Delta_{i} \equiv \Delta^{(1)}$, and find

$$
K_{i, j}(L)=\lim _{\Delta_{j} \rightarrow 0} \frac{e_{2}\left(\Delta^{(1)}, \Delta_{j}, L\right)}{\Delta^{(1)}} .
$$

In our approach, we calculate $e\left(\Delta_{i}^{(1)}, \Delta_{j}^{(k)}, L\right)$ for chains with up to $L_{C}=110$ atoms and for three values $\Delta_{j}^{(1,2,3)}$.
Then, we apply a fit of the linear function

$$
\epsilon(\Delta)=K_{i, j}(L) \Delta
$$

to the three points $\left(\Delta_{j}^{(k)}, e\left(\Delta^{(1)}, \Delta_{j}, L\right) / \Delta^{(1)}\right)$ and the origin. The slope defines $K_{i, j}(L)$.

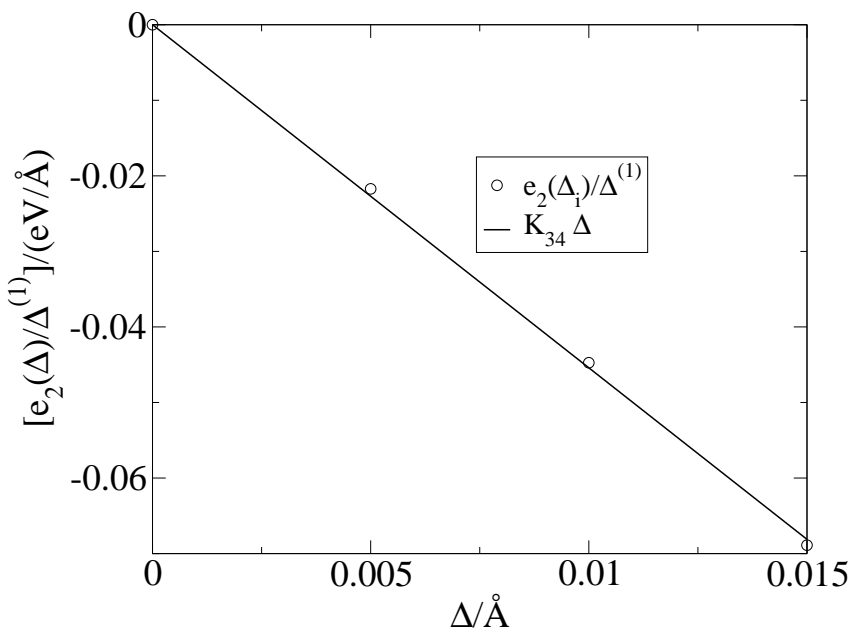

FIG. 18. Excitation energy for distortions of the first carbon atom in each unit cell in the $x$-direction in the presence of a distortion $\Delta^{(1)}$ in the $y$-direction, relative to the excitation energies of the individual distortions, eq. (B10), divided by $\Delta^{(1)}$ in units of eV/ $\AA$. Data are shown for the Hückel-Hubbard model on a zig-zag chain as a function of the distortion $\Delta$ in units of $\AA$ for open boundary conditions and the same parameter set as in Fig. 17 The straight line is a linear fit with $K_{34}=-4.54 \mathrm{eV} / \AA^{2}$.

As an example, in Fig. 18 we show the extrapolation of $\tilde{K}_{34}$ for the Hückel-Hubbard model for $U=6 \mathrm{eV}$ and $L=$ 32 unit cells on a zig-zag chain. It is seen again that the extrapolation is fairly stable and provides a reliable value for the 'spring constant' $\tilde{K}_{34}=\tilde{K}_{43}=-4.54 \mathrm{eV} / \AA^{2}$.

\section{c. Number of DMRG runs}

In this approach, we need a total number $N_{\text {run }}$ of ground-state DMRG calculations for fixed geometry. To calculate all elements of the symmetric $4 \times 4$ dynamical matrix, we need $N_{\text {run }}=4 \cdot 3+[(4 \cdot 3) / 2] \cdot 2=12+12=24$ DMRG calculations for each system size.

A more compact way to calculate $\tilde{K}_{i j}$ without an extrapolation in $\Delta$ would be to employ the second-order Hellman-Feynman theorem. Further information can be found in the supplemental material $\underline{\underline{20}}$ 
1 M. Born and K. Huang, Dynamical theory of crystal lattices, Clarendon Press, Oxford, 1956.

${ }^{2}$ H. Bilz and P. M. Schlag, Phonon dispersion relations in insulators, Springer, Berlin, 1979, vol. 10 Springer Series in Solid-State Sciences.

3 Ab initio calculation of phonon spectra, ed. J. T. Devreese, V. E. van Doren and P. E. van Camp, Plenum Press, New York, 1982.

4 S. Baroni, S. de Gironcoli, A. Dal Corso and P. Giannozzi, Rev. Mod. Phys., 2001, 73, 515-562.

5 J. Sólyom, Fundamentals of the Physics of Solids, Springer, Berlin, 2009, vol. 1.

6 B. Dorner, Coherent inelastic neutron scattering in lattice dynamics, Springer, Berlin, 1982, vol. 93 Springer Tracts in Modern Physics.

7 S. W. Lovesey, Theory of neutron scattering from condensed matter, Clarendon Press, Oxford, 2004, vol. $1 \mathrm{Nu}-$ clear scattering.

8 A. Furrer, J. Mesot and T. Strässle, Neutron scattering in condensed matter physics, World Scientific, Singapore, 2009, vol. 4 Series on neutron techniques and applications.

9 R. Claus, L. Merten and J. Brandmüller, Light Scattering by Phonon-Polaritons, Springer, Berlin, 1975, vol. 75 Springer Tracts in Modern Physics.

10 Infrared and Raman Spectroscopy, ed. B. Schrader, WileyVCH, Weinheim, 1995.

11 T. P. Devereaux and R. Hackl, Rev. Mod. Phys., 2007, 79, 175-233.

12 C. J. Pearson, W. Barford and R. J. Bursill, Phys. Rev. B, 2011, 83, 195105.

13 M. Capone, C. Castellani and M. Grilli, Advances in Condensed Matter Physics, 2010, 2010, Article ID 920860.

14 M. Hohenadler, F. F. Assaad and H. Fehske, Phys. Rev. Lett., 2012, 109, 116407.

15 H. Bakrim and C. Bourbonnais, Phys. Rev. B, 2015, 91, 085114

16 M. Weber, F. F. Assaad and M. Hohenadler, Phys. Rev. $B, 2015,91,235150$.

17 J. Sólyom, Fundamentals of the Physics of Solids, Springer, Berlin, 2009, vol. 2.

18 D. Baeriswyl, D. K. Campbell and S. Mazumdar, in Conjugated Conducting Polymers, ed. H. G. Kiess, Springer, Berlin, Heidelberg, 1992, vol. 102 Springer Series in Solid-
State Sciences, ch. 2, pp. 7-134.

19 W. Barford, Electronic and Optical Properties of Conjugated Polymers, Oxford Science Publications, Oxford, 2009.

20 See supplemental material.

21 L. Salem, Molecular Oribtal Theory of Conjugated Systems, Benjamin, New York, 1966.

22 S. R. White, Phys. Rev. Lett., 1992, 69, 2863-2866.

23 S. R. White, Phys. Rev. B, 1993, 48, 10345-10356.

24 U. Schollwöck, Rev. Mod. Phys., 2005, 77, 259-315.

25 U. Schollwöck, Annals of Physics, 2011, 326, 96 - 192.

26 S. Szalay, M. Pfeffer, V. Murg, G. Barcza, F. Verstraete, R. Schneider and Ö. Legeza, International Journal of Quantum Chemistry, 2015, 115, 1342.

27 H. Kuzmany, physica status solidi (b), 1980, 97, 521-531.

28 H. Takeuchi, Y. Furukawa, I. Harada and H. Shirakawa, The Journal of Chemical Physics, 1986, 84, 2882-2890.

29 H. Takeuchi, T. Arakawa, Y. Furukawa, I. Harada and H. Shirakawa, Journal of Molecular Structure, 1987, 158, 179-193.

30 E. Ehrenfreund, Z. Vardeny, O. Brafman and B. Horovitz, Phys. Rev. B, 1987, 36, 1535-1553.

31 M. Timar, G. Barcza, F. Gebhard, L. Veis and Ö. Legeza, Phys. Chem. Chem. Phys., 2016, 18, 18835-18845.

32 G. Barcza, W. Barford, F. Gebhard and Ö. Legeza, Phys. Rev. B, 2013, 87, 245116.

33 A. J. Heeger, S. Kivelson, J. R. Schrieffer and W.-P. Su, Rev. Mod. Phys., 1988, 60, 781-850.

34 W. Kohn, Phys. Rev. Lett., 1959, 2, 393-394.

35 A. Race, W. Barford and R. J. Bursill, Phys. Rev. B, 2003, 67, 245202.

36 Mathematica, Version 9.0, Wolfram Research, Inc., Champaign, 2012.

37 Ö. Legeza, J. Röder and B. A. Hess, Phys. Rev. B, 2003, 67, 125114 .

38 Ö. Legeza and J. Sólyom, Phys. Rev. B, 2004, 70, 205118.

39 H. Hellmann, Einführung in die Quantenchemie, Deuticke, Leipzig und Wien, 1937.

40 H. Hellmann, Einführung in die Quantenchemie, Springer, Berlin, 2015.

41 R. P. Feynman, Phys. Rev., 1939, 56, 340-343. 